\title{
Elaborate analysis and design of filter-bank-based sensing for wideband cognitive radios
}

\author{
Konstantinos Maliatsos*, Athanasios Adamis and Athanasios G Kanatas
}

\begin{abstract}
The successful operation of a cognitive radio system strongly depends on its ability to sense the radio environment. With the use of spectrum sensing algorithms, the cognitive radio is required to detect co-existing licensed primary transmissions and to protect them from interference. This paper focuses on filter-bank-based sensing and provides a solid theoretical background for the design of these detectors. Optimum detectors based on the Neyman-Pearson theorem are developed for uniform discrete Fourier transform (DFT) and modified DFT filter banks with root-Nyquist filters. The proposed sensing framework does not require frequency alignment between the filter bank of the sensor and the primary signal. Each wideband primary channel is spanned and monitored by several sensor subchannels that analyse it in narrowband signals. Filter-bank-based sensing is proved to be robust and efficient under coloured noise. Moreover, the performance of the weighted energy detector as a sensing technique is evaluated. Finally, based on the Locally Most Powerful and the Generalized Likelihood Ratio test, real-world sensing algorithms that do not require $a$ priori knowledge are proposed and tested.
\end{abstract}

Keywords: Filter bank; Spectrum sensing; Cognitive radio; Optimum detectors; GLRT; LMP

\section{Introduction}

Spectrum sensing has been brought into the center of research activities due to its application in the context of cognitive radio (CR) [1]. Cognitive radio and dynamic spectrum access have been identified as the means to maximize spectrum exploitation and efficiency. The cognitive radios share the available spectrum with a licensed primary system (PS) and have the responsibility not to adversely affect the PS user operation by causing interference. Spectrum sensing is used to identify and consequently avoid co-existing primary signals. Several spectrum sensing techniques have been derived and studied [2,3]. These algorithms present pros and cons concerning the need for a priori knowledge of PS signals features, the computational complexity, the robustness in channel variations and coloured noise etc.

In [4], the introduction of filter-bank-based sensing was made and further analysis was provided in [5]. The main

*Correspondence: kmaliat@unipi.gr

School of Information and Communications Technologies, Department of Digital Systems, University of Piraeus, M. Karaoli and A. Dimitriou 80, Piraeus 18534, Greece advantage of this technique lies in the fact that since the CR networks will use multicarrier modulations for transmission, the analysis filters that are intended for receiver operation, e.g. in OFDM-OQAM [6,7], in discrete wavelet multiTone [8] and in filtered multiTone [9], could also be used for sensing without extra computational workload. In [10], filter-bank-based physical layer design for CR systems was introduced, where simultaneous spectrum sensing and transmission may be possible using the filter bank. In [4] and [5], Fahrang-Boroujeny examined the filter bank operation as an estimator of the power Spectral density (PSD) incorporating a spectrum analyser in the receiver structure. A comparison in the performance of the estimation with the non-parametric multitaper method [11] was also made. It was confirmed that the use of a filter bank can similarly achieve remarkable spectral analysis with the use of a larger set of samples but much less computational complexity.

However, in $[4,5]$, no detectors were presented that could be practically used to identify primary emissions in a given band of interest. Since then, several studies were made regarding filter-bank-based sensing. Most studies

\section{Springer}

(c) 2014 Maliatsos et al.; licensee Springer. This is an Open Access article distributed under the terms of the Creative Commons Attribution License (http://creativecommons.org/licenses/by/2.0), which permits unrestricted use, distribution, and reproduction in any medium, provided the original work is properly credited. 
consider simplified models for the operation of the filter bank and do not attain optimality [12,13]. In [14], filter bank sensing is performed using data-aided feature detection achieving results in extremely low signal-tonoise ratio (SNR); however, the algorithm required knowledge for specific features of the PS signal. Some studies focus on the reduction of the computational workload [15-17], while others propose new filters suitable for sensing $[18,19]$. In addition, special issues have been addressed that concern the application of filter banks in spectrum sensing [20,21]. Moreover, in [22,23], efficient implementations of filter bank sensors are presented.

This study is motivated by the absence of strong theoretical description for filter-bank-based sensors. A variety of detectors are presented for uniform and modified discrete Fourier transform (uniform DFT-MDFT) filter banks and especially root-Nyquist filters. As in [14], filter banks are implemented using the polyphase structure in order to perform simultaneous parallel sensing on all subchannels; however, the proposed algorithms are not based on specific signal feature extraction. Optimality based on the Neyman-Pearson theorem [24] is achieved. Moreover, the designed sensors are based on the approach that there is no '1-1' matching of the receiver filters and the PS signal bandwidth. This is a common flaw among the majority of filter-bank-based sensing studies. Primary signals are in their vast majority wideband, while the CR filter bank has the objective to divide the monitored spectrum into narrowband channels. Therefore, in a common configuration, a primary channel is spanned and analysed by a number of CR filters. The use of multiple CR filters on a single PS transmission also eliminates the need for frequency alignment between the primary signal and the $\mathrm{CR}$ detector. An additional advantage is that the filter bank can partially exploit the radio channel frequency selectivity without the need for complicated equalization procedures on the primary signal. In [25] and [26], multiple CR subchannels are also used to span the primary signal. In the first study, a technique for SNR estimation of the PS signal is proposed using simple approximations, while in [26] the authors introduce a weighted energy detector scheme that is able to efficiently scan Bluetooth channels.

In Section 2, the used system model is presented. In Section 3, the Neyman-Pearson optimal detectors for uniform DFT banks are extracted, while in Section 4, the optimal detectors in the output of an OFDM-OQAM receiver (using an MDFT bank) are studied. In Section 5, the weighted energy detector for uniform DFT banks is analysed, and in Section 6, the extension of the filter bank sensing application in coloured noise is presented. It is noted that coloured noise has been identified [5] as a significant challenge for spectrum sensing applications. Finally in Section 7, practical implementations of the detectors - the Locally Most Powerful (LMP) test and the Generalized Likelihood Ratio Test (GLRT) - are proposed. Simulation results are presented in Section 8.

\section{System model}

A CR system is assumed that digitizes a large portion of spectrum containing $K$ channels of the PS. The wideband received signal is analysed by a bank of $M>K$ filters that span the whole digitized bandwidth. Consequently, each PS channel is analysed by $\left[L=M_{/ K}\right\rceil$ filters. A graphical representation of a 32-channel filter bank that spans the bandwidth that contains four PS channels is presented in Figure 1. In the specific example, the output from eight subchannels can be used to extract the decision on whether a PS channel is occupied or not.

Given the fact that the wideband CR receiver should optimize the use of the limited computational resources, the analysis focuses on uniform DFT [27] filter banks with downsampling at the Nyquist rate. The well-known computationally efficient polyphase structure can be assumed. The type-1 polyphase representation of an finite impulse response (FIR) filter is the following [28]:

$$
h_{i}^{(1)}(n)=h(M n+i), \quad H(z)=\sum_{i=0}^{M-1} z^{-i} H_{i}^{(1)}\left(z^{M}\right)
$$

where the subscript indicates the $i$ th polyphase filter path, while the superscript defines the type of polyphase representation. Analysis is also expanded in filter banks used by OFDM-OQAM systems. The specific filters present similarities with the cosine-modulated filter banks and their digitally equivalent representation is the MDFT [29] filter banks [29]. Ideally, a digital implementation of an OFDMOQAM system will use MDFT filter banks with perfect reconstruction (PR) properties. In many cases, the PR property is sacrificed for better time-frequency localization; however, these filters can also be expressed with the MDFT structure and it is highly desired to approximate the behaviour of MDFT-PR. Two equivalent structures of an OFDM-OQAM receiver are presented in Figure 2 (where normalization and phase correction coefficients are arbitrarily set since they do not affect the results of the specific study). The two filter structures are equivalent for $D$ filter coefficients where $\alpha=\left\lfloor\frac{D-1}{M / 2}\right\rfloor=2 \alpha^{\prime}$, i.e., $\alpha$ is even. Nevertheless, the selection of odd $\alpha$ does not affect the MDFT-filter bank-based spectrum sensing results.

Moreover, the assumed prototype filter is real and symmetric ensuring linear phase response. Deeper analysis is performed for filter banks using a root-Nyquist prototype filter [30]. The root-Nyquist filters are typically used for pulse shaping in radio communication systems due to the 


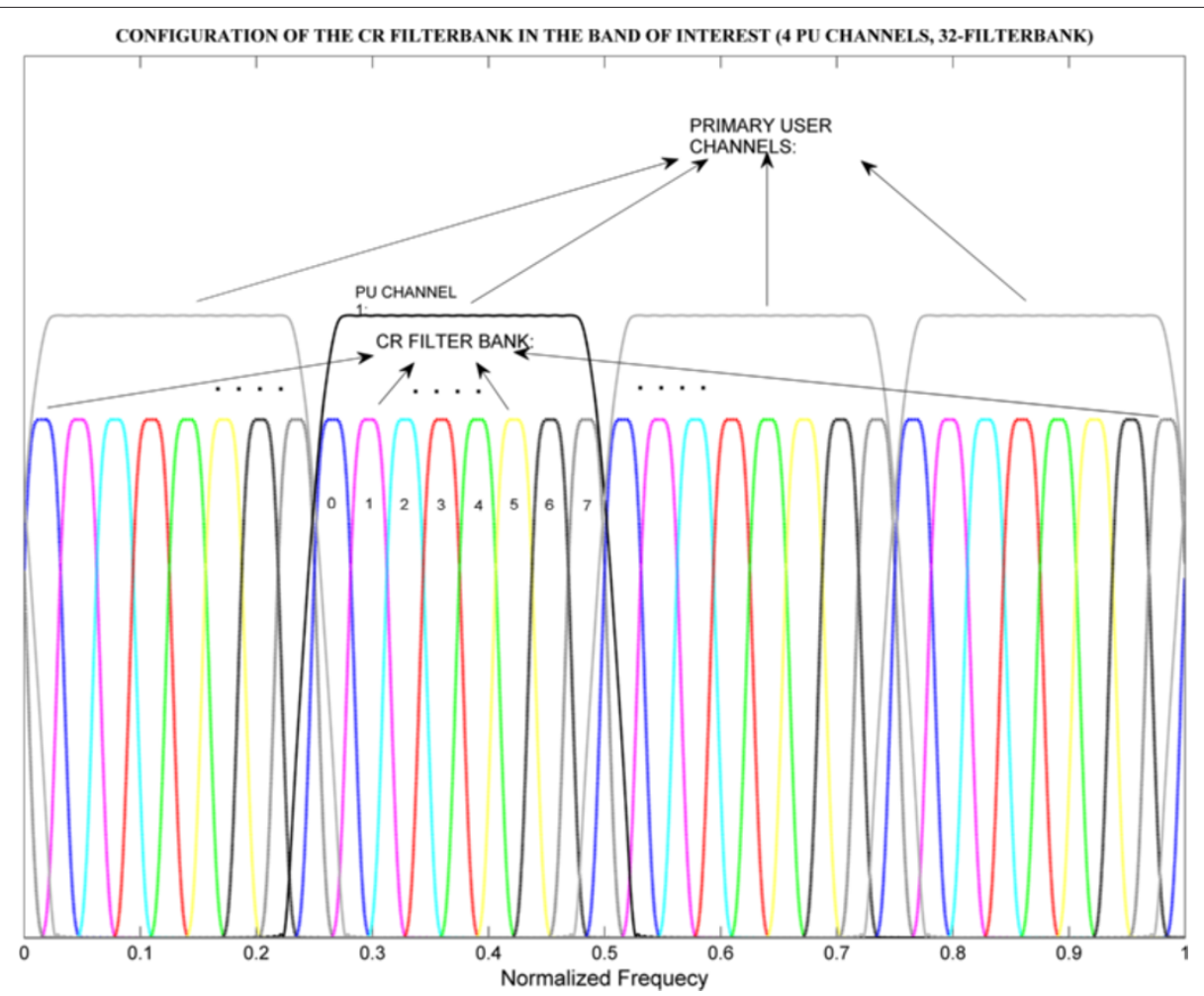

Figure 1 Filter bank configuration example for $M=32$ in a bandwidth containing four PS channels.

InterSymbol Interference (ISI)-free transmission properties. It is noted that the necessary and sufficient condition for MDFT-PR filters suitable for OFDM-OQAM also leads to root-Nyquist filters [29].

The $n$th time sample of the received signal from the output of the A/D converter is represented by $r_{n}$, while $y_{n}^{(i)}$ represents the output of the $i$ th filter. It is assumed that the filter impulse response is normalized so that the mean input and output signal powers remain constant, if the incoming signal is contained in the specific filter passband. Thus, $h_{u}=p_{u} / \sqrt{\sum_{u=-\infty}^{\infty}\left|p_{u}\right|^{2}}$, where $p_{u}$ is the FIR

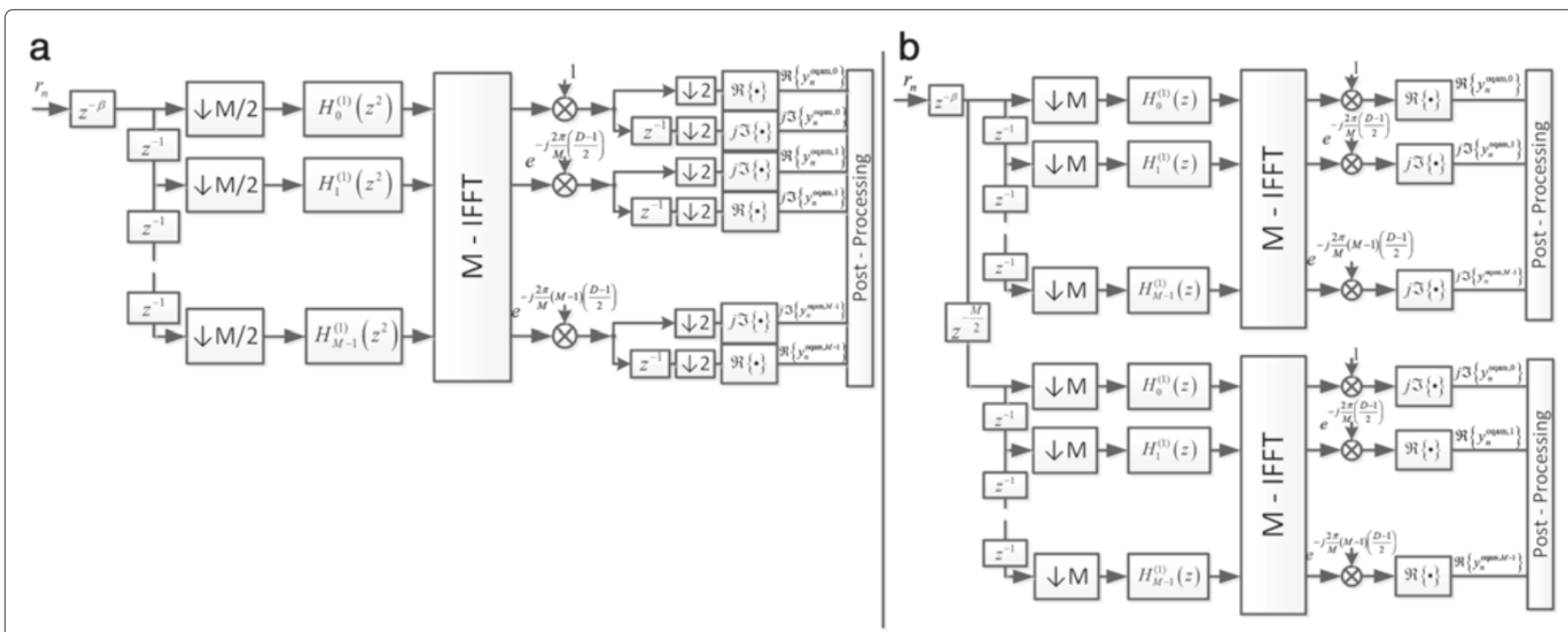

Figure 2 Two OFDM-OQAM receiver filter structures. (a) Conventional implementation and (b) parallel uniform DFT bank implementation. 
filter prototype. Using a non-casual filter representation, the Nyquist ISI-free condition is expressed by

$$
g_{n}=\sum_{u=-\infty}^{\infty} h_{u} h_{n-u}=\left\{\begin{array}{l}
1, \quad n=0 \\
0, \quad n=l M, l \neq 0
\end{array}\right.
$$

The presented theoretical analysis assumes that the transmitted PS signal $s_{n}$ is circular white Gaussian random variable with mean power at the receiver (over a flat channel) $\sigma_{s}^{2}$. This assumption is accurate for orthogonal frequency division multiplexing (OFDM) signals, but it is questionable for single carrier signals. However, since the signal passes through a cascade of filters and transceiver impairments, each signal sample is the result of linear combinations and shifts that includes a large number of random variables. Thus, a generalized version of the Central Limit Theorem can be invoked to justify the Gaussian assumption for the PS signal. The incoming signal at the $\mathrm{CR}$ receiver for given PS transmissions under frequency selective channels is provided by

$$
r_{n}=\sum_{k=0}^{K-1} \zeta_{k} \sum_{u=0}^{\infty} c_{u}^{(k)} s_{n-u}^{(k)}+w_{n}
$$

where the superscripts indicate the transmitted signal at the $k$ th primary channel, and the $\zeta_{k}$ coefficient is equal to 0 if the $k$ th channel is free, or 1 if the $k$ th channel is occupied. Filter $c_{u}^{(k)}$ represents the radio channel impulse response for the $k$ th PS channel. The formulated detection problem concerns the use of the available information from multiple filter bank outputs in the decision of whether a primary channel is occupied or not. Therefore, the binary decision problem for the $k$ th primary channel is expressed as

$$
\begin{aligned}
& \mathcal{H}_{0}: \quad \zeta_{k}=0 \rightarrow r_{n}^{(k)}=w_{n} \\
& \mathcal{H}_{1}: \quad \zeta_{k}=1 \rightarrow r_{n}^{(k)}=\sum_{u=0}^{\infty} c_{u}^{(k)} s_{n-u}^{(k)}+w_{n}
\end{aligned}
$$

The observation vector that contains the information used for the decision is a set of $N$ samples (depending on the sensing duration) for each of the $L$ filters that span the primary channel under investigation. The following vectors are defined:

$$
\mathbf{y}_{l}=\left[\begin{array}{llll}
y_{n}^{(l)} & y_{n-1}^{(l)} & \ldots & y_{n-N+1}^{(l)}
\end{array}\right]^{\mathrm{T}}
$$

with

$$
y_{n}^{(l)}=\sum_{u=-\infty}^{\infty} h_{u}^{(l)} r_{M n-u}
$$

where $h_{u}^{(l)}$ is the impulse response for the lth subchannel of the filter bank. In (6), it is assumed that the signal is maximally decimated at the output. Without loss of generality, $L$ is assumed even for presentation purposes. No additional information on primary signal features and specifications is assumed. The next step is the definition of the detectors.

\section{Neyman-Pearson optimal detectors for maximally decimated signals}

The energy detector is the simplest and most common spectrum sensing technique with low complexity and minimum knowledge regarding the primary system. The energy detector can be seen as a special case of filter-bankbased sensing with $M=1$ and $K=1$. It is proved that for zero-mean white Gaussian input, the energy detector is optimal according to the Neyman-Pearson theorem [24] for known PS signal power $\sigma_{s}^{2}$ and additive white Gaussian noise (AWGN) with $\sigma_{w}^{2}$.

$$
T=\sum_{n=0}^{N-1}|y[n]|^{2} \underset{\mathcal{H}_{0}}{\stackrel{\mathcal{H}_{1}}{\gtrless}} \gamma
$$

The metric $T$ follows a scaled $\chi^{2}$ distribution as the sum of squares of white Gaussian zero-mean random variables [31] with $2 N$ degrees of freedom. It is proved that $T$ follows the distributions below:

$$
T=\left\{\begin{array}{c}
\frac{\sigma_{w}^{2}}{2} \chi_{2 N}^{2} \text { If } \mathcal{H}_{0}, \\
\frac{\sigma_{w}^{2}+\sigma_{s}^{2}}{2} \chi_{2 N}^{2} \text { If } \mathcal{H}_{1}
\end{array}\right.
$$

In this paper, the energy detector is used as a reference for the evaluation of the extracted algorithms.

When referring to maximally decimated signals, it is considered that the rate of the signals at the filter output is reduced to the minimum as it is defined by the Nyquist sampling theorem. This means that the specific detector operates at the output of a uniform DFT filter bank (or the output of one of the parallel banks in an OFDM-OQAM system before the real-imaginary separation - Figure $2 b$ ). The sensing mechanism can be considered as a vector detection problem with vector observations from $L$ different sensors. Assuming that the $k_{0}$ th primary channel is observed, the output from the filter paths with indexes $k_{0} L+l, l=0, \ldots, L-1$ is collected.

The first step is to define a single observation vector with the use of a reordering technique for the selected vectors. For reasons that will be cleared out at the next steps, the following reordering is selected:

- The observation vectors are divided in two groups:

- Group 0 contains the even subchannels.

- Group 1 contains the odd subchannels.

- Spatial reordering (or row rollout [24]) is performed (choosing first the group 0 vector). Therefore,

$$
\begin{aligned}
& \mathbf{y}=\left[\left(\mathbf{y}^{(0)}\right)^{\mathrm{H}} \mid\left(\mathbf{y}^{(1)}\right)^{\mathrm{H}}\right]^{\mathrm{H}}
\end{aligned}
$$

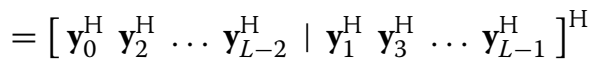


where $\mathbf{y}_{l}$ is given in (5). For simplicity reasons, the common term $k_{0} L$ of the vector subscripts was omitted. Subvectors $\mathbf{y}_{i}$ represent the observation vector per filter path, i.e. per subchannel.

The signal PSD under $\mathcal{H}_{1}$ after filtering and before decimation is given by

$$
\begin{aligned}
S_{y_{i} y_{i}}(f) & =S_{r r}(f)\left|H\left(f-f_{i}\right)\right|^{2} \\
& =\left(\sum_{k=0}^{K-1}\left|C^{(k)}(f)\right|^{2} S_{s s}^{(k)}(f)+S_{w w}(f)\right)\left|H\left(f-f_{i}\right)\right|^{2}
\end{aligned}
$$

where $C^{(k)}(f)$ is the radio channel transfer function for the PS signal that occupies the $k$ th subchannel and $S_{s s}^{(k)}(f), S_{r r}(f)$ the respective PSDs for $s_{n}$ and $r_{n}$ respectively. As in [4], it is considered that the receiver filters are adequately narrowband. This assumption is valid for OFDM-OQAM systems since by design the filters should divide the whole bandwidth in subchannels where the radio channel frequency response can be considered approximately flat (since otherwise computationally cumbersome equalization methods would be required). Therefore, the following approximation can be considered:

$$
\begin{aligned}
S_{y_{i} y_{i}}(f) & \simeq\left(\left|C\left(f_{i}\right)\right|^{2} S_{s s}(f)+S_{w w}(f)\right)\left|H\left(f-f_{i}\right)\right|^{2} \\
& =\left(\left|c_{i}\right|^{2} \sigma_{s}^{2}+\sigma_{w}^{2}\right)\left|H\left(f-f_{i}\right)\right|^{2}
\end{aligned}
$$

where the PSDs for the white Gaussian signal and noise were used. The $c_{i}$ coefficient is a measure of the radio channel effect for the specific subchannel obtained by the radio channel Fourier transform. Although this study assumes flat radio channel per filter subchannel, this requirement is not very strict. During simulation and detector evaluation, the radio channel models did not provide strictly flat radio channels per filter. Nevertheless, under realistic channel conditions, no significant fluctuations from the assumed optimum performance under the flat channel assumption was observed. Moreover, a better approximation of the $c_{i}$ coefficients can be made with the use of a mean value of the frequency selective channel transfer function for each subchannel filter. More specifically,

$$
\left|c_{i}\right|^{2}=\frac{1}{\mathrm{BW}} \int_{-\infty}^{\infty}\left|C(f) H\left(f-f_{i}\right)\right|^{2} d f
$$

where BW is the total bandwidth. From (11), it is concluded that with the use of the narrowband filter, the input signal for each subchannel can be considered an AWGN random variable with $\mathbf{r}_{i} \sim \mathcal{N}\left(0,\left(\left|c_{i}\right|^{2} \sigma_{s}^{2}+\sigma_{w}^{2}\right) \mathbf{I}_{N}\right)$, despite the radio channel effects. Under $\mathcal{H}_{0}$, the input signal is also AWGN with $\mathbf{r}_{i} \sim \mathcal{N}\left(0, \sigma_{w}^{2} \mathbf{I}_{N}\right)$. In Figure 3, two examples of Rayleigh channels with exponential power delay profiles (PDP) are presented. The first example (maximum delay spread 12 samples and exponent $\lambda=1$ ) presents a quite accurate flat narrowband channel approximation. In the second case (maximum delay spread 24 samples with exponent 0.5), frequency selectivity is apparent in the passband. However, the flat approximation is used in both cases with higher expected deviation from the ideal for the second example.

Generally, for large $M$ and adequately small number of filter coefficients, the filter transition bandwidth is not quite sharp. However, it can be assumed that the subchannels of the filter bank provide sufficient attenuation at the passband so that the following approximation stands:

$$
\begin{aligned}
& i=0, \ldots, M-1 \\
& H\left(f-f_{i}\right) H^{*}\left(f+f_{i+l}\right) \simeq 0, \quad i+l=0, \ldots, M-1 \\
& l \neq-1,0,1
\end{aligned}
$$

In (13) it is claimed that the outputs from two filter paths that are not directly adjacent are uncorrelated and under the Gaussian assumption independent. According to the Neyman-Pearson theorem, the detector that optimizes the probability of detection $P_{\mathrm{D}}$ for given probability of false alarm $P_{\mathrm{FA}}$ is provided by the likelihood ratio:

$$
\frac{f\left(\mathbf{y} / \mathcal{H}_{1}\right)}{f\left(\mathbf{y} / \mathcal{H}_{0}\right)} \stackrel{\mathcal{H}_{1}}{\gtrless} \theta
$$

where $f$ is the probability density function (PDF) for the random variable (operand '/ indicates a conditional probability). Threshold $\theta$ is selected in order to achieve the

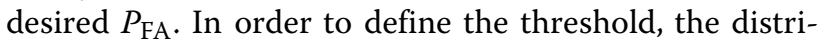
butions of the vector random variables, as well as the distributions of the final detection metric must be defined. Based on the initial assumptions, $\mathbf{y}$ is a multidimensional Gaussian variable. The first task is to determine its covariance matrix. Similarly to the energy detector, the SNR, or equivalently the signal and noise powers, should be known. More specifically, the investigated detector should be aware of the SNR per subchannel that is given by $\gamma_{i}=$ $\frac{\left|c_{i}\right|^{2} \sigma_{s}^{2}}{\sigma_{w}^{2}}$. Initially, this information is assumed known. In a real-world design of the detector, an estimation procedure is performed before or during the detection (Section 7).

\subsection{Distribution under $\mathcal{H}_{0}$}

The determination of the covariance matrix for the specified block vector with spatial reordering is made using the following relationship: 


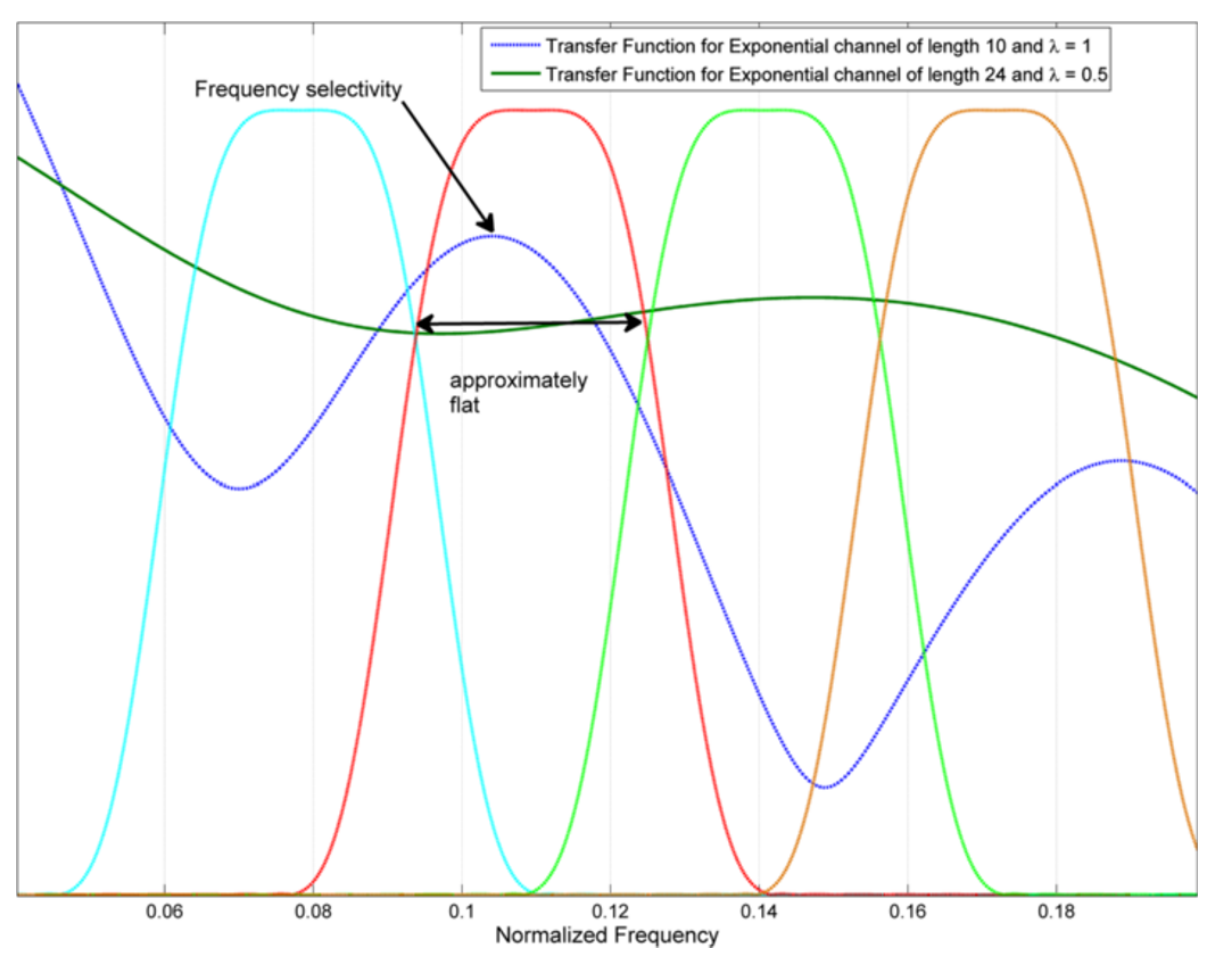

Figure 3 Examples of radio channel transfer functions per subchannel for $M=32$ and Rayleigh channels with exponential power delay profiles.

$$
\mathrm{E}\left(\mathbf{y} \mathbf{y}^{\mathrm{H}}\right)=\left[\begin{array}{c:c}
\mathbf{E}_{00} & \mathbf{E}_{01} \\
\hdashline-- & -- \\
\mathbf{E}_{10} & \mathbf{E}_{11}
\end{array}\right]
$$

where,

$$
\mathbf{E}_{i j}=\left[\begin{array}{cccc}
\mathrm{E}\left(\mathbf{y}_{i} \mathbf{y}_{j}^{\mathrm{H}}\right) & \mathrm{E}\left(\mathbf{y}_{i} \mathbf{y}_{j+2}^{\mathrm{H}}\right) & \ldots & \mathrm{E}\left(\mathbf{y}_{i} \mathbf{y}_{j+L-2}^{\mathrm{H}}\right) \\
\mathrm{E}\left(\mathbf{y}_{i+2} \mathbf{y}_{j}^{\mathrm{H}}\right) & \mathrm{E}\left(\mathbf{y}_{i+2} \mathbf{y}_{j+2}^{\mathrm{H}}\right) & & \mathrm{E}\left(\mathbf{y}_{i+2} \mathbf{y}_{j+L-2}^{\mathrm{H}}\right) \\
\vdots & \ddots & \ddots & \vdots \\
\mathrm{E}\left(\mathbf{y}_{i+L-2} \mathbf{y}_{j}^{\mathrm{H}}\right) & \mathrm{E}\left(\mathbf{y}_{i+L-2} \mathbf{y}_{j+2}^{\mathrm{H}}\right) & \ldots & \mathrm{E}\left(\mathbf{y}_{i+L-2} \mathbf{y}_{j+L-2}^{\mathrm{H}}\right)
\end{array}\right]
$$

Initially, in order to quantify the effect of the filter bank on the variables, the input signal variance for all subchannels is assumed to be $\sigma^{2}=1$. The covariance of the maximally decimated signal for each subchannel separately is given by [4]

$$
\begin{aligned}
\rho_{y_{i} y_{i}}(n) & =\sum_{u=-\infty}^{\infty} h_{u} e^{2 \pi j \frac{u i}{M}} h_{M n+u} e^{-2 \pi j \frac{u i+M n i}{M}} \\
& =\sum_{u=-\infty}^{\infty} h_{u} h_{M n+u}=g_{M n}
\end{aligned}
$$

For a root-Nyquist filter and according to (2), the result is equal to unity for $n=0$ and zero otherwise. Therefore, the output samples of each subchannel are uncorrelated despite the filter bank.

$$
\mathrm{E}\left(\mathbf{y}_{i} \mathbf{y}_{i}^{\mathrm{H}}\right)=\left[\begin{array}{ccc}
\rho_{y_{i} y_{i}}(0) & \ldots & \rho_{y_{i} y_{i}}(N-1) \\
\vdots & \ddots & \\
\rho_{y_{i} y_{i}}(-N+1) & & \rho_{y_{i} y_{i}}(0)
\end{array}\right]=\mathbf{I}_{N}
$$

Based on the aforementioned assumption (13), the output vectors from not direct-adjacent filter paths are uncorrelated and therefore,

$$
\mathrm{E}\left(\mathbf{y}_{i} \mathbf{y}_{i+l}^{\mathrm{H}}\right)=\mathbf{0}_{N}, \quad l \neq-1,0,1
$$

It remains to calculate the cross-correlation $[\mathbf{R}]_{m, n}=$ $\rho_{y_{i} y_{i+l}}(m-n)(m, n=1 \ldots N)$ between the output samples of adjacent filters $(l=-1,1)$.

The elements of $\mathbf{R}$ are calculated with the following formula:

$$
\begin{aligned}
& {[\mathbf{R}]_{k, m}=\mathrm{E}\left(y_{n}^{(i)} y_{n+m-k}^{(i+l) *}\right)=\mathrm{E}\left(y_{n}^{(i)} y_{n+\varepsilon}^{(i+l) *}\right)=} \\
& \sum_{u=-\infty}^{\infty} \sum_{p=-\infty}^{\infty} h_{u} h_{p} e^{2 \pi j \frac{i u}{M}} e^{-2 \pi j \frac{(i+l) p}{M}} \mathrm{E}\left(r_{M n-u} r_{M n-p+M \varepsilon}^{*}\right)
\end{aligned}
$$


where $m-k=\varepsilon$. For zero-mean white Gaussian input with $\sigma^{2}=1$, the following result is extracted:

$$
\begin{aligned}
{[\mathbf{R}]_{k, m} } & =\delta_{p+M \varepsilon-u} \sum_{u=-\infty}^{\infty} \sum_{p=-\infty}^{\infty} h_{u} h_{p} e^{2 \pi j \frac{i u}{M}} e^{-2 \pi j \frac{(i+l) p}{M}} \\
& =\sum_{u=-\infty}^{\infty} h_{u} h_{u-M \varepsilon} e^{2 \pi j \frac{i u}{M}} e^{-2 \pi j \frac{(i+l)(u-M \varepsilon)}{M}} \\
& =\sum_{u=-\infty}^{\infty} h_{u} e^{-2 \pi j \frac{l u}{M}} h_{u-M \varepsilon}
\end{aligned}
$$

which can be proved to be equal to the following:

$$
\begin{aligned}
& {[\mathbf{R}]_{k, m}=g_{M(m-k)}^{\cos } \triangleq \sum_{u=-\infty}^{\infty} h_{u} \cos \left(\frac{2 \pi u}{M}\right) h_{u+M(k-m)}} \\
& \mathbf{R}=\left[\begin{array}{cccc}
g_{0}^{\cos } & g_{M}^{\cos } & \ldots & g_{M(N-1)}^{\cos } \\
g_{-M}^{\cos } & g_{0}^{\cos } & & \\
\vdots & & \ddots & \vdots \\
g_{-M(N-1)}^{\cos } & \cdots & g_{0}^{\cos }
\end{array}\right]
\end{aligned}
$$

The matrix $\mathbf{R}$ is real. It is noted that filters are expressed with a non-casual representation in order to exploit symmetries in a simple way. As a next step, the following $\frac{L N}{2} \times \frac{L N}{2}$ low triangular block matrix is defined:

$$
\mathbf{P} \triangleq \underbrace{\left[\begin{array}{ccccc}
\mathbf{R} & \mathbf{0} & & \ldots & \mathbf{0} \\
\mathbf{R} & \mathbf{R} & \mathbf{0} & & \\
\mathbf{0} & \mathbf{R} & \mathbf{R} & \ddots & \\
\vdots & & \ddots & \mathbf{0} \\
\mathbf{0} & \mathbf{0} & \mathbf{R} & \mathbf{R}
\end{array}\right]}_{L / 2}
$$

Based on the definition of (23) and using (18), (19) and (22), the produced result is given by

$$
\mathrm{E}\left(\mathbf{y y}^{\mathrm{H}} / r \sim \mathcal{N}(0,1)\right) \triangleq \mathbf{C}=\left[\begin{array}{ccc}
\mathbf{I}_{L N} & \mid & \mathbf{P} \\
- & - & - \\
\mathbf{P}^{\mathrm{T}} & \mid & \mathbf{I}_{L N / 2}
\end{array}\right]
$$

The definition of the $L N \times L N$ matrix leads directly to the extraction of the covariance matrix for variable $y$ given that the primary channel is free $\left(\mathcal{H}_{0}\right)$. It is straightforward to claim that

$$
\mathcal{H}_{0}: \mathbf{C}_{y}=\mathbf{C}_{w}=\sigma_{w}^{2} \mathbf{C} \Rightarrow \mathbf{y} / \mathcal{H}_{0} \sim \mathcal{N}\left(\mathbf{0}, \sigma_{w}^{2} \mathbf{C}\right)
$$

The fact that $\mathbf{C}$ is expressed as a $2 \times 2$ block matrix with identity matrices in the block diagonal justifies the selection of the specific reordering since the computations are now quite simplified. More specifically, the inverse of $\mathbf{C}$ is given by ([32])

$$
\mathbf{C}^{-1}=\left[\begin{array}{ccc}
\left(\mathbf{I}_{L N / 2}-\mathbf{P} \mathbf{P}^{\mathrm{T}}\right)^{-1} & \mid & -\mathbf{P}\left(\mathbf{I}_{L N / 2}-\mathbf{P}^{\mathrm{T}} \mathbf{P}\right)^{-1} \\
- & - & - \\
-\mathbf{P}^{\mathrm{T}}\left(\mathbf{I}_{L N / 2}-\mathbf{P} \mathbf{P}^{\mathrm{T}}\right)^{-1} & \mid & \left(\mathbf{I}_{L N / 2}-\mathbf{P}^{\mathrm{T}} \mathbf{P}\right)^{-1}
\end{array}\right]
$$

An important feature is that the determinant of $\mathbf{C}$ can be easily calculated using $|\mathbf{C}|=\left|\mathbf{I}_{L N / 2}-\mathbf{P P}^{\mathrm{T}}\right|$ [33], since the matrix is real and symmetric. In addition, the conditional probabilities between groups 0 (even filter paths) and 1 (odd filter paths) are extracted:

$\mathbf{y} \sim \mathcal{N}\left(\mathbf{0}, \mathbf{C}_{w}\right) \Rightarrow\left\{\begin{array}{c}\mathbf{y}^{(0)} / \mathbf{y}^{(1)} \sim \mathcal{N}\left(\mathbf{P y}^{(1)}, \sigma_{w}^{2}\left(\mathbf{I}-\mathbf{P P}^{\mathrm{T}}\right)\right) \\ \mathbf{y}^{(1)} / \mathbf{y}^{(0)} \sim \mathcal{N}\left(\mathbf{P}^{\mathrm{T}} \mathbf{y}^{(0)}, \sigma_{w}^{2}\left(\mathbf{I}-\mathbf{P}^{\mathrm{T}} \mathbf{P}\right)\right)\end{array}\right.$

\subsection{Distribution under $\mathcal{H}_{1}$}

According to the initial assumptions, the primary signal $s_{n}$ follows the normal distribution with mean power $\sigma_{s}^{2}$. Initially, a noiseless channel is assumed. Due to the radio channel and based on the approximation in (11), the input signal power per subchannel is given by the multiplication of the mean signal power with a radio channel coefficient, i.e. the assumed input per subchannel is given by $r_{n}^{(i)}=$ $c_{i} s_{n}$ and the mean input signal power per subchannel is $\mathrm{E}\left(\left|r_{n}^{(i)}\right|^{2}\right)=\mathrm{E}\left(\left|c_{i} s_{n}\right|^{2}\right)=\left|c_{i}\right|^{2} \sigma_{s}^{2}$. The calculation of the covariance matrix of the observation vector is made using the procedure of the previous paragraph with the introduction of the radio channel coefficient effects. Since the result presented in (18) has been computed for standard zero-mean Gaussian input $\left(\sigma^{2}=1\right)$, the autocovariance matrix for the output of the $i$ th filter path, when the input is given by $r_{n}^{(i)}=c_{i} s_{n}$, is provided by

$$
\mathrm{E}\left(\mathbf{y}_{i} \mathbf{y}_{i}^{\mathrm{H}}\right)=\left|c_{i}\right|^{2} \sigma_{s}^{2} \mathbf{I}_{N}
$$

The cross-covariance submatrix between filter outputs of non-directly adjacent paths is once again zero. For directly adjacent filter paths, computation is performed as in (20) with the inclusion of the channel coefficients.

$$
\begin{aligned}
& {[\mathbf{R}]_{k, m}=\mathrm{E}\left(y_{n}^{(i)} y_{n+m-k}^{(i+l) *}\right)=\mathrm{E}\left(y_{n}^{(i)} y_{n+\varepsilon}^{(i+l) *}\right)=} \\
& \sum_{u=-\infty}^{\infty} \sum_{p=-\infty}^{\infty} h_{u} h_{p} e^{2 \pi j \frac{i u}{M}} e^{-2 \pi j \frac{(i+l) p}{M}} \mathrm{E}\left(c_{i} s_{M n-u} c_{i+l}^{*} s_{M n-p+M \varepsilon}^{*}\right)
\end{aligned}
$$

In order to avoid complex coefficients, since there is no simple method to estimate their phase, the following rationale is considered: A complex zero-mean Gaussian variable can also be seen as a pair of two random variables: (a) the Rayleigh amplitude $\left|s_{n}\right| \sim$ Rayleigh $\left(\sigma_{s}^{2}\right)$ and (b) the uniform in $[0,2 \pi)$ phase $\varphi_{s_{n}} \sim \mathcal{U}(0,2 \pi)$. After the 
application of the channel coefficient on the signal, the considered input of the $i$ th filter is given by

$$
r_{n}^{(i)}=c_{i}\left|s_{n}\right| e^{j \varphi_{s_{n}}}=\left|c_{i}\right|\left|s_{n}\right| e^{j \varphi_{s_{n}}+\varphi_{c_{i}}}
$$

However, the radio-channel-induced phase shifts due to the uniform phase distribution and periodicity will not cause any variation in the signal phase distribution and therefore:

$$
\varphi_{s_{n}}+\varphi_{c_{i}} \sim \mathcal{U}(0,2 \pi) \Rightarrow s_{n}^{\prime}=\left|s_{n}\right| e^{j\left(\varphi_{s_{n}}+\varphi_{c_{i}}\right)} \sim \mathcal{N}\left(0, \sigma_{s}^{2}\right)
$$

From (31) and (30) it is extracted that

$$
r_{n}^{(i)} \sim \mathcal{N}\left(0,\left|c_{i}\right|^{2} \sigma_{s}^{2}\right)
$$

Using (32) and (29) similarly to (21), it is concluded that

$$
\mathrm{E}\left(\mathbf{y}_{i} \mathbf{y}_{i+l}^{\mathrm{H}}\right)=\sigma_{s}^{2}\left|c_{i}\right|\left|c_{i+l}\right| \mathbf{R}
$$

The diagonal matrix $\boldsymbol{\Sigma}$ is defined:

$$
\boldsymbol{\Sigma}=\left[\begin{array}{cccccccc}
\left|c_{0}\right| \mathbf{I}_{N} & \mathbf{0} & \mathbf{0} & & \ldots & & & \mathbf{0} \\
\mathbf{0} & \left|c_{2}\right| \mathbf{I}_{N} & \mathbf{0} & \mathbf{0} & & & & \\
\mathbf{0} & \ddots & \ddots & \ddots & \ddots & & & \\
& \ddots & \mathbf{0} & \left|c_{L-2}\right| \mathbf{I}_{N} & \mathbf{0} & \mathbf{0} & & \\
\vdots & & \mathbf{0} & \mathbf{0} & \left|c_{1}\right| \mathbf{I}_{N} & \mathbf{0} & \ddots & \\
& & & \mathbf{0} & \mathbf{0} & \left|c_{3}\right| \mathbf{I}_{N} & \mathbf{0} & \mathbf{0} \\
& & & & \ddots & \ddots & \ddots & \mathbf{0} \\
\mathbf{0} & & & & & \mathbf{0} & \mathbf{0} & \left|c_{L-1}\right| \mathbf{I}_{N}
\end{array}\right]
$$

where the diagonal elements contain the channel coefficients according to the adopted reordering. Based on the definition of $\boldsymbol{\Sigma}$, it is concluded that under $\mathcal{H}_{1}$ with no noise, the received signal covariance matrix is given by

$$
\mathbf{C}_{s}=\sigma_{s}^{2} \boldsymbol{\Sigma} \mathbf{C} \boldsymbol{\Sigma}
$$

Let us now assume that AWGN with mean power $\sigma_{w}^{2}$ is also present during reception. In this case, the input for the $i$ th subchannel assuming that the primary signal $s_{n}$ follows the zero-mean Gaussian distribution with mean power $\sigma_{s}^{2}$ is given by

$$
r_{n}^{(i)}=c_{i} s_{n}+w_{n}
$$

where $w_{n}$ is the noise component with $w_{n} \sim \mathcal{N}\left(0, \sigma_{w}^{2}\right)$. It was proved for a noiseless signal in (32) that $c_{i} s_{n} \sim$ $\mathcal{N}\left(\mathbf{0},\left|c_{i}\right|^{2} \sigma_{s}^{2}\right)$. The input signal for the $i$ th subchannel $r_{n}^{(i)}$ is given by the sum of two zero-mean Gaussian variables with variances $\left|c_{i}\right|^{2} \sigma_{s}^{2}$ and $\sigma_{w}^{2}$. Based on the properties of the Gaussian distribution [24] and given that signal and noise are independent and uncorrelated random variables, $r_{n}^{(i)}$ will also follow the zero-mean Gaussian distribution and its variance will be provided by the sum of the variances of the two random variables (signal and noise). Therefore,

$$
r_{n}^{(i)} \sim \mathcal{N}\left(0,\left|c_{i}\right|^{2} \sigma_{s}^{2}+\sigma_{w}^{2}\right)
$$

If $z_{n}$ is a zero-mean Gaussian variable with $\sigma^{2}=1$, then the variable $\left(\sqrt{\left|c_{i}\right|^{2} \sigma_{s}^{2}+\sigma_{w}^{2}}\right) z_{n}$ follows the same distribution with $r_{n}^{(i)}$ and it can be used for the calculation of the autocovariance and cross-covariance matrices. Similarly to (28) and (29), it is proved that

$$
\mathrm{E}\left(\mathbf{y}_{i} \mathbf{y}_{i}^{\mathrm{H}}\right)=\left(\left|c_{i}\right|^{2} \sigma_{s}^{2}+\sigma_{w}^{2}\right) \mathbf{I}_{N}
$$

and for $l= \pm 1$,

$$
\begin{aligned}
\mathrm{E}\left(y_{n}^{(i)} y_{n+m-k}^{(i+l) *}\right)= & \sum_{u=-\infty}^{\infty} \sum_{p=-\infty}^{\infty} h_{u} h_{p} e^{2 \pi j \frac{i u}{M}} e^{-2 \pi j \frac{(i+l) p}{M}} \\
& \times \mathrm{E}\left(r_{M n-u} r_{M n-p+M \varepsilon}^{*}\right) \\
= & \sum_{u=-\infty}^{\infty} \sum_{p=-\infty}^{\infty} h_{u} h_{p} e^{2 \pi j \frac{i u}{M}} e^{-2 \pi j \frac{(i+l) p}{M}} \times \\
& \times \mathrm{E}\left(\left(\sqrt{\left|c_{i}\right|^{2} \sigma_{s}^{2}+\sigma_{w}^{2}}\right) z_{M n-u}\right. \\
& \left.\times\left(\sqrt{\left|c_{i+l}\right|^{2} \sigma_{s}^{2}+\sigma_{w}^{2}}\right) z_{M n-p+M \varepsilon}^{*}\right) \\
= & \left(\sqrt{\left|c_{i}\right|^{2} \sigma_{s}^{2}+\sigma_{w}^{2}}\right)\left(\sqrt{\left|c_{i+l}\right|^{2} \sigma_{s}^{2}+\sigma_{w}^{2}}\right)[\mathbf{R}]_{k, m}
\end{aligned}
$$

which concludes that $\mathrm{E}\left(\mathbf{y}_{i} \mathbf{y}_{i+l}^{\mathrm{H}}\right)=\left(\sqrt{\left|c_{i}\right|^{2} \sigma_{s}^{2}+\sigma_{w}^{2}}\right)$ $\left(\sqrt{\left|c_{i+l}\right|^{2} \sigma_{s}^{2}+\sigma_{w}^{2}}\right) \mathbf{R}$. The combination of the results from (38) and (39) are now used to extract the correlation matrix for vector $\mathbf{y}$ defined in (9). It is proved that

$$
\mathbf{C}_{y}=\boldsymbol{\Sigma}_{y} \mathbf{C} \boldsymbol{\Sigma}_{y}, \leftrightarrow \mathbf{C}_{y}^{-1}=\boldsymbol{\Sigma}_{y}^{-1} \mathbf{C}^{-1} \boldsymbol{\Sigma}_{y}^{-1}
$$

where $\Sigma_{y}$ is the diagonal matrix containing the factors $\left(\sqrt{\left|c_{i}\right|^{2} \sigma_{s}^{2}+\sigma_{w}^{2}}\right)$.

$$
\boldsymbol{\Sigma}_{y}=\left(\sigma_{s}^{2} \boldsymbol{\Sigma}^{2}+\sigma_{w}^{2} \mathbf{I}_{L N}\right)^{1 / 2}
$$

Thus, based on the Gaussian distribution properties, since the observation vector under $\mathcal{H}_{1}$ is a linear transformation of the zero-mean Gaussian input and its covariance matrix is given by (40), it follows the distribution of (42):

$$
\mathbf{y} / \mathcal{H}_{1} \sim \mathcal{N}\left(\mathbf{0},\left(\sigma_{s}^{2} \boldsymbol{\Sigma}^{2}+\sigma_{w}^{2} \mathbf{I}_{L N}\right)^{1 / 2} \mathbf{C}\left(\sigma_{s}^{2} \boldsymbol{\Sigma}^{2}+\sigma_{w}^{2} \mathbf{I}_{L N}\right)^{1 / 2}\right)
$$

If the known (or estimated) quantity is the SNR $\gamma_{i}$, the channel coefficients can be calculated from $\left|c_{i}\right|^{2}=$ $\gamma_{i} \sigma_{w / \sigma_{s}^{2}}^{2}$ 


\subsection{The detector metric}

In order to define the detector, starting from (14), the loglikelihood relationship is formed:

$$
\begin{gathered}
\ln \left(\left|\mathbf{C}_{w}\right|\right)-\ln \left(\left|\mathbf{C}_{y}\right|\right)+\mathbf{y}^{\mathrm{H}} \mathbf{C}_{w}^{-1} \mathbf{y}-\mathbf{y}^{\mathrm{H}} \mathbf{C}_{y}^{-1} \mathbf{y} \underset{\mathcal{H}_{0}}{\stackrel{\mathcal{H}_{1}}{\gtrless}} \ln (\theta) \Rightarrow \\
\mathbf{y}^{\mathrm{H}}\left(\mathbf{C}_{w}^{-1}-\mathbf{C}_{y}^{-1}\right) \mathbf{y} \underset{\mathcal{H}_{0}}{\stackrel{\mathcal{H}_{1}}{\gtrless}} \theta^{\prime}=\ln (\theta)+\ln \left(\left|\mathbf{C}_{y}\right|\right)-\ln \left(\left|\mathbf{C}_{w}\right|\right)
\end{gathered}
$$

Therefore, the metric that will be used for the decision is provided by

$$
T(\mathbf{y})=\mathbf{y}^{\mathrm{H}}\left(\mathbf{C}_{w}^{-1}-\mathbf{C}_{y}^{-1}\right) \mathbf{y}
$$

\subsection{Metric distribution under $\mathcal{H}_{0}$}

Initially, the eigenvalue decomposition of matrix $\mathbf{C}=$ $\mathbf{U} \boldsymbol{\Lambda} \mathbf{U}^{\mathrm{H}}$ is performed and since $\mathbf{C}$ is hermitian $\mathbf{U} \mathbf{U}^{\mathrm{H}}=$ $\mathbf{U}^{\mathrm{H}} \mathbf{U}=\mathbf{I}$. With the use of the eigenvalues and eigenvectors, an interim variable can be defined:

$$
\mathbf{z}=\frac{1}{\sigma_{w}} \boldsymbol{\Lambda}^{-1 / 2} \mathbf{U}^{\mathrm{H}} \mathbf{y}
$$

It is simple to prove that this variable decorrelates the samples of vector $\mathbf{y}$ and that $\mathbf{z} \sim \mathcal{N}\left(\mathbf{0}, \mathbf{I}_{L N}\right)$. Thus, the metric can be written as

$$
\begin{aligned}
\mathbf{y}^{\mathrm{H}}\left(\mathbf{C}_{w}^{-1}-\mathbf{C}_{y}^{-1}\right) \mathbf{y} \Rightarrow & \mathbf{z}^{\mathrm{H}} \sigma_{w} \boldsymbol{\Lambda}^{1 / 2} \mathbf{U}^{\mathrm{H}}\left(1 / \sigma_{w}^{2} \mathbf{C}^{-1}-\boldsymbol{\Sigma}_{y}{ }^{-1} \mathbf{C}^{-1} \boldsymbol{\Sigma}_{y}{ }^{-1}\right) \\
& \times \mathbf{U} \boldsymbol{\Lambda}^{1 / 2} \sigma_{w} \mathbf{z} \\
= & \mathbf{z}^{\mathrm{H}}\left(\mathbf{I}-\sigma_{w}^{2} \boldsymbol{\Lambda}^{1 / 2} \mathbf{U}^{\mathrm{H}} \boldsymbol{\Sigma}_{y}{ }^{-1} \mathbf{C}^{-1} \boldsymbol{\Sigma}_{y}{ }^{-1} \mathbf{U} \boldsymbol{\Lambda}^{1 / 2}\right) \mathbf{z}
\end{aligned}
$$

In order to calculate the eigenvalues of the matrix that appears at the quadratic form of the metric, the definition of the characteristic polynomial is used:

$$
\begin{aligned}
\operatorname{det} & \left(\mathbf{I}-\sigma_{w}^{2} \boldsymbol{\Lambda}^{1 / 2} \mathbf{U}^{\mathrm{H}} \boldsymbol{\Sigma}_{y}{ }^{-1} \mathbf{C}^{-1} \boldsymbol{\Sigma}_{y}{ }^{-1} \mathbf{U} \boldsymbol{\Lambda}^{1 / 2}-\chi \mathbf{I}\right) \\
& =\operatorname{det}\left(\sigma_{w}^{2} \boldsymbol{\Lambda}^{1 / 2} \mathbf{U}^{\mathrm{H}} \boldsymbol{\Sigma}_{y}{ }^{-1} \mathbf{C}^{-1} \boldsymbol{\Sigma}_{y}{ }^{-1} \mathbf{U} \boldsymbol{\Lambda}^{1 / 2}+(\chi-1) \mathbf{I}\right) \\
& =\operatorname{det}(\boldsymbol{\Lambda}) \operatorname{det}\left(\sigma_{w}^{2} \boldsymbol{\Sigma}_{y}{ }^{-1} \mathbf{C}^{-1} \boldsymbol{\Sigma}_{y}{ }^{-1}+(\chi-1) \mathbf{C}^{-1}\right)=0
\end{aligned}
$$

The determinant of $\boldsymbol{\Lambda}$ can be omitted. In order to move forward, the approach described below is followed:

- The inverse of $\mathbf{C}^{-1}$ is also an hermitian matrix.

- Thus, the matrix $\mathbf{V}=\sigma_{w}^{2} \boldsymbol{\Sigma}_{y}{ }^{-1} \mathbf{C}^{-1} \boldsymbol{\Sigma}_{y}{ }^{-1}+(\chi-1) \mathbf{C}^{-1}$ can be seen as the sum of two covariance matrices of multidimensional Gaussian variables. It is known from probability theory [34] that if two random variables are independent, the covariance matrix of their sum is equal to the sum of the covariance matrices. Let us assume a random variable that follows the distribution $\mathcal{N}\left(0, \sigma_{w}^{2} \Sigma_{y}{ }^{-2}\right)$ and a second random variable that follows the distribution $\mathcal{N}(0,(\chi-1) \mathbf{I})$. In addition, the two variables are considered independent. It is assumed that the variables are added and applied to the selected filter bank. Then, the covariance matrix of their sum at the output of the bank will be provided by matrix $\mathbf{V}$.

- However, for independent Gaussian variables a different approach can be used that produces equivalent results. The sum of two independent zeromean Gaussian random variables is also a zero-mean Gaussian random variable. The variance of the sum is equal with the sum of variances [34]. Therefore, instead of considering the sum of two independent random variables applied to the filter bank, it is equivalent to assume a single random variable that follows the distribution $\mathcal{N}\left(0, \sigma_{w}^{2} \Sigma_{y}^{-2}+(\chi-1) \mathbf{I}\right)$.

- Based on the second approach, the covariance matrix at the output of the filter will be given by

$\mathbf{W}=\left(\sigma_{w}^{2} \boldsymbol{\Sigma}_{y}{ }^{-2}+(\chi-1) \mathbf{I}\right)^{\frac{1}{2}} \mathbf{C}^{-1}\left(\sigma_{w}^{2} \boldsymbol{\Sigma}_{y}{ }^{-2}+(\chi-1) \mathbf{I}\right)^{\frac{1}{2}}$

The extraction of the covariance matrix in this case is performed using the same procedure that was followed in (39), (40) and (41).

- Since the two procedures are equivalent for Gaussian random variables, it is expected that the equivalence will also stand between the covariance matrices $\mathbf{V}$ and $\mathbf{W}$ and therefore they can be used interchangeably. The followed approach is valid, since the eigenvalues extracted by $\mathbf{V}$ are applied on the Gaussian random variable $\mathbf{z} \sim \mathcal{N}\left(\mathbf{0}, \mathbf{I}_{L N}\right)$. In addition, the validity of the procedure was verified with extended tests.

- Thus, instead of calculating the determinant of $\mathbf{V}$, we proceed using matrix W. From (48) and given the fact that the determinant of $\mathbf{C}$ is non-zero (since $\mathbf{C}$ is invertible), it is extracted that

$\operatorname{det}\left(\sigma_{w}^{2} \Sigma_{y}^{-2}+(\chi-1) \mathbf{I}\right)=0 \Rightarrow \chi_{i}=1-\frac{\sigma_{w}^{2}}{\sigma_{s}^{2}\left|c_{i}\right|^{2}+\sigma_{w}^{2}}$

- With the use of $\mathbf{V}$-W equivalence, the metric matrix is considered to have $L$ discrete eigenvalues with $N$ degrees of multiplicity each. The eigenvector matrix does not play any role in the procedure and it does not need to be calculated. Since $\mathbf{U}$ is unitary, the random variable $\tilde{\mathbf{z}}=\mathbf{U}^{\mathrm{H}} \mathbf{z}$ will follow the same distribution as z. Therefore, the metric under $\mathcal{H}_{0}$ is given by

$$
T\left(\mathbf{y} / \mathcal{H}_{0}\right)=\sum_{l=0}^{L-1} \sum_{n=0}^{N-1} \frac{\sigma_{s}^{2}\left|c_{l}\right|^{2}}{\sigma_{s}^{2}\left|c_{l}\right|^{2}+\sigma_{w}^{2}}\left|\tilde{z}_{n+l N}\right|^{2}
$$

- In order to verify the validity of the predescribed equivalence, extended tests were performed for 
various filters and radio channels. The eigenvalues of matrix $\mathbf{V}$ were numerically extracted and used directly for metric calculation. It was observed that in all cases, the eigenvalues of $\mathbf{V}$ and $\mathbf{W}$ were very similar. However, the most important observation was that they produced, as expected, identical distributions when applied to $\mathbf{z}$ for metric calculation. Therefore, the equivalence was also verified via simulations.

In (50), each $\tilde{z}_{i}$ element follows the standard complex Gaussian distribution (unit variance). Consequently,

$$
2\left|\tilde{z}_{n+l N}\right|^{2} \sim \chi_{2}^{2}
$$

The chi-square distribution can be considered a special case of Gamma distribution. In fact, if a random variable $\omega$ follows the $\chi_{2}^{2}$ distribution, for a positive constant $\beta$, the variable $\beta \omega$ follows the gamma distribution with parameters $(1,2 \beta)[35]$. Therefore,

$$
\frac{\sigma_{s}^{2}\left|c_{l}\right|^{2}}{\sigma_{s}^{2}\left|c_{l}\right|^{2}+\sigma_{w}^{2}}\left|\tilde{z}_{n+l N}\right|^{2} \sim \mathcal{G}\left(1, \frac{\sigma_{s}^{2}\left|c_{l}\right|^{2}}{\sigma_{s}^{2}\left|c_{l}\right|^{2}+\sigma_{w}^{2}}\right)
$$

where $\mathcal{G}$ denotes the gamma distribution. In order to calculate the metric distribution, the characteristic function for $\mathcal{G}$ is used. Moreover, since the addends in (50) are independent, the characteristic function of the total metric will be given by the product of the functions of the individual variables. Thus,

$$
\varphi_{T\left(\mathbf{y} / \mathcal{H}_{0}\right)}(\tau)=\prod_{n=0}^{L-1}\left(\frac{1}{1-j \frac{\sigma_{s}^{2}\left|c_{l}\right|^{2}}{\sigma_{s}^{2}\left|c_{l}\right|^{2}+\sigma_{w}^{2}} \tau}\right)^{N}
$$

The PDF is given by the inverse Fourier of the characteristic function at point $-t$.

$$
f_{T\left(\mathbf{y} / \mathcal{H}_{0}\right)}(t)=\mathcal{F}_{-t}^{-1}\left\{\prod_{n=0}^{L-1}\left(\frac{1}{1-j \frac{\sigma_{s}^{2}\left|c_{l}\right|^{2}}{\sigma_{s}^{2}\left|c_{l}\right|^{2}+\sigma_{w}^{2}} \tau}\right)^{N}\right\}
$$

The calculation of the analytical solution is not a straightforward task because of the fact that each eigenvalue is repeated $N$ times. An analytical solution can be achieved using the distribution provided in [36] where the positive definite matrix of [36] is the identity matrix. The metric distribution is given by

$$
\begin{aligned}
f_{T(\mathbf{y})}\left(t>0 / \mathcal{H}_{0}\right)= & \prod_{i=1}^{L-1}\left(\frac{\lambda_{\min }^{\mathrm{udft} / \mathcal{H}_{0}}}{\lambda_{l}^{\mathrm{udft} / \mathcal{H}_{0}}}\right)^{N} \\
& \times \sum_{k=0}^{\infty} \frac{d_{k} t^{L N+k-1} e^{-\left(\frac{t}{\lambda_{\min }^{\mathrm{udft} / \mathcal{H}_{0}}}\right)}}{\left(\lambda_{\min }^{\mathrm{udft} / \mathcal{H}_{0}}\right)^{L N+k} \Gamma(L N+k)}
\end{aligned}
$$

where coefficients $d_{k}$ are calculated iteratively using

$$
\begin{gathered}
d_{0}=1, \\
d_{k+1}=\frac{N}{k+1} \sum_{i=1}^{k+1}\left[\sum_{l=1}^{L-1}\left(1-\frac{\lambda_{\min }^{\mathrm{udft} / H_{0}}}{\lambda_{l}^{\mathrm{udft} / H_{0}}}\right)^{i}\right] d_{k+1-i}, \quad k=0,1,2 \ldots
\end{gathered}
$$

and the $\lambda_{i} \mathrm{~s}$ are the $L$ discrete eigenvalues, thus,

$$
\lambda_{i}^{\mathrm{udft} / \mathcal{H}_{0}}=\frac{\sigma_{s}^{2}\left|c_{i}\right|^{2}}{\sigma_{w}^{2}+\sigma_{s}^{2}\left|c_{i}\right|^{2}}
$$

According to the Neyman-Pearson theorem, the threshold is calculated from the probability of false alarm $P_{\text {FA }}$ under $\mathcal{H}_{0}$ through the complimentary cumulative distribution function (CDF). The following relationship is used [36]:

$$
P_{\mathrm{FA}}=1-\prod_{i=1}^{L-1}\left(\frac{\lambda_{\min }^{\mathrm{udft} / \mathcal{H}_{0}}}{\lambda_{l}^{\mathrm{udft} / \mathcal{H}_{0}}}\right)^{N} \sum_{k=0}^{\infty} d_{k}\left(\frac{\Gamma\left(L N+k, \frac{\theta}{\lambda_{l}^{\mathrm{udft} / \mathcal{H}_{0}}}\right)}{\Gamma(L N+k)}\right)
$$

Threshold $\theta$ is calculated for a given $P_{\mathrm{FA}}$ with numerical inversion of the function.

The main problem regarding the use of (55), (56) and (58) in a sensing algorithm is the infinite sum. In a practical implementation, the truncation of the infinite sum is unavoidable. In order to calculate the number of needed addends (or equivalently $d_{k}$ coefficients) that will provide a sufficient approximation of the distribution, the following simulation procedure was followed. A large set of 50,000 channels with exponential PDP was produced. The exponential PDP is given by

$$
\mathrm{PDP}=\exp (-\lambda n), \text { for } n=0,1 \ldots, C_{\max }
$$

During the simulation, the used PDP parameters were $\lambda=$ $0.7, C_{\max }=48$. The $c_{i}$ coefficients were calculated using (12) and the eigenvalues $\lambda_{i}^{\mathrm{udft} / \mathcal{H}_{0}}$ were determined using (57). It was concluded that the number of the necessary $d_{k}$ coefficients depends on the spread of the extracted eigenvalues. This means that if the standard deviation of the $L$ eigenvalues is small, then the approximation converges fast to the distribution of (55); otherwise, a very large number of $d_{k}$ coefficients should be calculated. In order to extract an empirical rule for the number of needed coefficients, the ratio of the maximum eigenvalue $\lambda_{\max }^{\mathrm{udt} / \mathcal{H}_{0}}$ over the minimum eigenvalue $\lambda_{\mathrm{min}}^{\mathrm{udft} / \mathcal{H}_{0}}$ was used as a measure of the eigenvalue spread. For each channel, the values 
in (55) and (56) were calculated iteratively as $k$ was continuously increasing. In each step, numerical integration of the estimated PDF was performed. When the integration result becomes larger than 0.9999 , it is assumed that the approximation has converged to the desired distribution.

In Figure 4, the simulation results are presented. It can be seen that in the vast majority of cases, less than 1,000 coefficients are needed for successful convergence. The prediction bounds of the linear fit (using the minimum mean square error (MMSE) technique) for 99.99\% confidence level are also provided in Figure 4. It was estimated that based on the simulation, the upper prediction bound is described by the following straight line:

$$
y=25.26 x+300.3
$$

Therefore, if the number of coefficients is estimated using (60), then there will be $99.99 \%$ probability that the approximated distribution has successfully converged. It is noted that with the use of the described procedure, a very accurate estimate of the $P_{\mathrm{FA}}$ from (58) is achieved with absolute error less than $10^{-5}$ for $P_{\mathrm{FA}}>0.25$.

In order to avoid the need for an approximation of an infinite sum, a method of numerical integration of the characteristic function can be used. During this study, a simple fast Fourier transform (FFT)-based algorithm that performs the integration was developed. Let us assume that the energy of the characteristic function is concen-

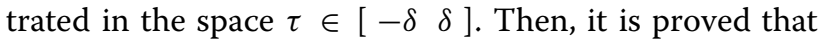
(54) can be accurately approximated by

$$
\hat{f}_{T(\mathbf{y})}\left(t_{k}=\frac{\pi k}{\delta} / \mathcal{H}_{0}\right)=\frac{\delta e^{j \pi k}}{\pi N^{\prime}} \operatorname{FFT}\left[\varphi_{T\left(\mathbf{y} / \mathcal{H}_{0}\right)}(n \Delta \tau-\delta)\right]_{N^{\prime}}
$$

The FFT-length $N^{\prime}$ can be found as an integer with $N^{\prime} \Delta \tau=\tau_{\max }$ with $\tau_{\max }$ a value where the PDF is expected to be practically zero and $\Delta \tau=\frac{1}{2 \delta}$. Therefore, fast Fourier transforms can be used to approximate with high accuracy the distribution under investigation. The complimentary CDF can be approximated with the trapezoidal numerical integration technique. Thus, for a threshold $\theta^{\prime}=\frac{\pi k_{0}}{\delta}$, the $P_{\text {FA }}$ is given by

$$
P_{\mathrm{FA}} \simeq \sum_{n=0}^{k_{0}} \frac{\pi}{\delta}\left(\frac{\hat{f}_{T(\mathbf{y})}\left(\frac{\pi k}{\delta} / \mathcal{H}_{0}\right)+\hat{f}_{T(\mathbf{y})}\left(\frac{\pi(k+1)}{\delta} / \mathcal{H}_{0}\right)}{2}\right)
$$

Given the fact that based on the aforementioned analysis, the selected threshold $\theta^{\prime}$ is also discrete, an approximation of the $P_{\mathrm{FA}}$ for any threshold can be calculated using linear interpolation.

\subsection{Metric distribution under $\mathcal{H}_{1}$}

Under $\mathcal{H}_{1}$, the interim variable can be defined as

$$
\mathbf{z}=\boldsymbol{\Lambda}^{-1 / 2} \mathbf{U}^{\mathrm{H}} \boldsymbol{\Sigma}_{y}^{-1} \mathbf{y}
$$

In this case, the determinant that provides the eigenvalues is given by

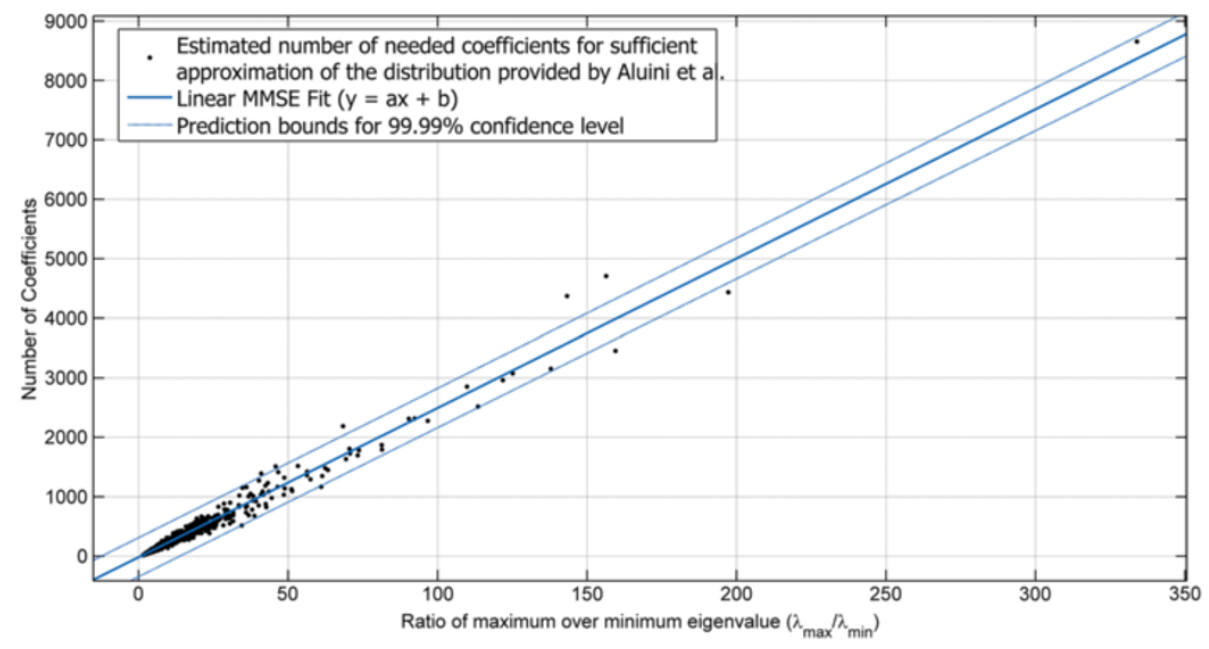

Figure 4 Simulation results of the number of coefficients for distribution approximation. 


$$
\begin{aligned}
\operatorname{det} & \left(\frac{\boldsymbol{\Sigma}_{y} \mathbf{C}^{-1} \boldsymbol{\Sigma}_{y}}{\sigma_{w}^{2}}-(\chi+1) \mathbf{C}^{-1}\right) \\
& =\operatorname{det}\left(\left(\frac{\boldsymbol{\Sigma}_{y}^{2}}{\sigma_{w}^{2}}-(\chi+1) \mathbf{I}\right)^{\frac{1}{2}} \mathbf{C}^{-1}\left(\frac{\boldsymbol{\Sigma}_{y}^{2}}{\sigma_{w}^{2}}-(\chi+1) \mathbf{I}\right)^{\frac{1}{2}}\right)=0
\end{aligned}
$$

which leads to $\chi_{i}=\frac{\sigma_{s}^{2}\left|c_{i}\right|^{2}}{\sigma_{w}^{2}}$.

The calculation of the metric distribution under $\mathcal{H}_{1}$ can be made with the same techniques as before:

- Using the analytical solution (55) and (56), where the used eigenvalues are $\lambda_{i}^{\mathrm{udft} / \mathcal{H}_{1}}=\frac{\sigma_{s}^{2}\left|c_{i}\right|^{2}}{\sigma_{w}^{2}}$

- With FFT-based approximation of the PDF from the characteristic function given by

$$
\varphi_{T\left(\mathbf{y} / \mathcal{H}_{1}\right)}(\tau)=\prod_{n=0}^{L-1}\left(1-j \frac{\sigma_{s}^{2}\left|c_{i}\right|^{2}}{\sigma_{w}^{2}} \tau\right)^{-N}
$$

After the extraction of the metric distribution under $\mathcal{H}_{1}$ and for a given threshold, the probability of correct detection $P_{\mathrm{D}}$ can be calculated. Using the analytical solution, the following formula is derived [36]:

$P_{\mathrm{D}}=1-\prod_{i=1}^{L-1}\left(\frac{\lambda_{\min }^{\mathrm{udft} / \mathcal{H}_{1}}}{\lambda_{l}^{\mathrm{udft} / \mathcal{H}_{1}}}\right)^{N} \sum_{k=0}^{\infty} d_{k}\left(\frac{\Gamma\left(L N+k, \frac{\theta^{\prime}}{\lambda_{l}^{\mathrm{udt} / \mathcal{H}_{1}}}\right)}{\Gamma(L N+k)}\right)$

When the truncation of the infinite sum is done using the ratio of the maximum over the minimum eigenvalue and the empirical formula of (60), then the approximation of the $P_{\mathrm{D}}$ value is extremely accurate (absolute error less than $\left.10^{-4}\right)$.

\section{Detector at the OFDM-OQAM receiver output}

The candidacy of OFDM-OQAM as a modulation technique for CR system leads inevitably to the need for a detector design for such systems. As mentioned in Section 2, the OFDM-OQAM systems can be assumed to use an MDFT filter bank structured as the block diagrams in Figure 2. The two structures are equivalent and can be used to implement the filtering unit of an OFDM-OQAM demodulator. In order to design the detector, the samples after the real/imaginary separation blocks are collected. At this point, the signal has practically returned to the Nyquist sampling rate. The second structure is selected, which contains two similar parallel uniform DFT banks with maximally decimated output in order to simplify the mathematical formulation of the problem. The two banks are operating with relevant time offset of $M / 2$ symbols. The signal that is led to the detector can be defined as

$$
\begin{aligned}
\mathbf{y}_{2 i}^{\text {oqam }} & =\Re\left(\mathbf{y}_{2 i}^{(\mathrm{A})}\right)+j \Re\left(\mathbf{y}_{2 i}^{(\mathrm{B})}\right) \\
\mathbf{y}_{2 i+1}^{\mathrm{oqam}} & =j \Im\left(\mathbf{y}_{2 i+1}^{\mathrm{(A)}}\right)+\Re\left(\mathbf{y}_{2 i+1}^{(\mathrm{B})}\right) \\
i & =0,1 . . M / 2
\end{aligned}
$$

The superscript indicates whether the specific signal is extracted by the first upper bank (A) or the second lower bank (B). Initially, an examination is performed to verify that there is no correlation between the real and imaginary part of the signal. The real/imaginary separation blocks can be expressed as simple linear relationships:

$$
\begin{aligned}
& \Re\left(\mathbf{y}_{i}^{(\mathrm{X})}\right)=\left[\frac{\mathbf{y}_{i}^{(\mathrm{X})}+\left(\mathbf{y}_{i}^{(\mathrm{X})}\right)^{*}}{2}\right], \Im\left(\mathbf{y}_{i}^{(\mathrm{X})}\right) \\
& =-j\left[\frac{\mathbf{y}_{i}^{(\mathrm{X})}-\left(\mathbf{y}_{i}^{(\mathrm{X})}\right)^{*}}{2}\right] \\
& \mathrm{X}=[\mathrm{A}, \mathrm{B}], i=0,1 \ldots M-1
\end{aligned}
$$

First, the autocorrelation matrix for each subchannel is calculated. It is noted that the presented analysis is provided for the even channels, but the exact same results are extracted for the odd subchannels with permutation of the $\Re(\bullet)$ and $\Im(\bullet)$ operators:

$$
\begin{aligned}
{\left[\mathrm{E}\left(\mathbf{y}_{i}^{\mathrm{oqam}}\left(\mathbf{y}_{i}^{\mathrm{oqam}}\right)^{\mathrm{H}}\right)\right]_{k, m}=} & \mathrm{E}\left(y_{n}^{\mathrm{oqam}, i}\left(y_{n+m-k}^{\mathrm{oqam}, i}\right)^{*}\right) \\
= & \mathrm{E}\left(y_{n}^{\mathrm{oqam}, i}\left(y_{n+\varepsilon}^{\mathrm{oqam}, i}\right)^{*}\right) \\
= & \mathrm{E}\left(\Re\left(y_{n}^{(\mathrm{A}, i)}\right) \Re\left(y_{n+\varepsilon}^{(\mathrm{A}, i)}\right)\right) \\
& +\mathrm{E}\left(\Im\left(y_{n}^{(\mathrm{B}, i)}\right) \Im\left(y_{n+\varepsilon}^{(\mathrm{B}, i)}\right)\right) \\
& +j \mathrm{E}\left(\Re\left(y_{n+\varepsilon}^{(\mathrm{A}, i)}\right) \Im\left(y_{n}^{(\mathrm{B}, i)}\right)\right) \\
& -j \mathrm{E}\left(\Re\left(y_{n}^{(\mathrm{A}, i)}\right) \Im\left(y_{n+\varepsilon}^{(\mathrm{B}, i)}\right)\right) \\
& \text { where }(m-k=\varepsilon)
\end{aligned}
$$

Assuming a circular white Gaussian input, the following result is extracted from (18) and (19):

$$
\begin{aligned}
\mathrm{E}\left(\Re\left(y_{n}^{(\mathrm{A})}\right) \Re\left(y_{n+\varepsilon}^{(\mathrm{A})}\right)\right) & =\frac{1}{2} \sigma_{x, i}^{2} \delta(\varepsilon) \\
\mathrm{E}\left(\Im\left(y_{n}^{(\mathrm{B})}\right) \Im\left(y_{n+\varepsilon}^{(\mathrm{B})}\right)\right) & =\frac{1}{2} \sigma_{x, i}^{2} \delta(\varepsilon) \\
\text { with } \sigma_{x, i}^{2} & =\left\{\begin{array}{c}
\sigma_{w}^{2}, \text { for } \mathcal{H}_{0} \\
\left|c_{i}\right|^{2} \sigma_{s}^{2}+\sigma_{w}^{2}, \text { for } \mathcal{H}_{1}
\end{array}\right.
\end{aligned}
$$

The investigation of the correlation of the imaginary parts in (69) leads to 


$$
\begin{aligned}
& \mathrm{E}\left(\Re\left(y_{n+\varepsilon}^{(\mathrm{A}, i)}\right) \Im\left(y_{n}^{(\mathrm{B}, i)}\right)\right)=\mathrm{E}\left(-j\left(\frac{y_{n+\varepsilon}^{(\mathrm{A}, i)}+\left(y_{n+\varepsilon}^{(\mathrm{A}, i)}\right)^{*}}{2}\right)\left(\frac{y_{n}^{(\mathrm{B}, i)}-\left(y_{n}^{(\mathrm{B}, i)}\right)^{*}}{2}\right)\right) \\
& =\frac{j}{4}\left(\mathrm{E}\left(y_{n+\varepsilon}^{(\mathrm{A}, i)}\right) \mathrm{E}\left(\left(y_{n}^{(\mathrm{B}, i)}\right)^{*}\right)\right)-\frac{j}{4}\left(\mathrm{E}\left(\left(y_{n+\varepsilon}^{(\mathrm{A}, i)}\right)^{*}\right) \mathrm{E}\left(y_{n}^{(\mathrm{B}, i)}\right)\right) \\
& =\frac{j}{4}\left(\sum_{u=-\infty}^{\infty} \sum_{p=-\infty}^{\infty} h_{u}^{*} h_{p} e^{2 \pi j \frac{i(p-u)}{M}} \mathrm{E}\left(x_{M n-u-\frac{M}{2}}^{*} x_{M n+M \varepsilon-p}\right)\right. \\
& \left.-\sum_{u=-\infty}^{\infty} \sum_{p=-\infty}^{\infty} h_{u}^{*} h_{p} e^{2 \pi j \frac{i(p-u)}{M}} \mathrm{E}\left(x_{M n+M \varepsilon-u^{x}}^{*}{ }_{M n-p-\frac{M}{2}}\right)\right) \\
& =\frac{j}{4}\left(\sum_{p=-\infty}^{\infty} h_{p} h_{p-M \varepsilon-\frac{M}{2}} e^{j \pi i}-\sum_{u=-\infty}^{\infty} h_{u} h_{u-M \varepsilon-\frac{M}{2}} e^{-j \pi i}\right)=0
\end{aligned}
$$

In (71), all the terms containing $\mathrm{E}\left(x_{n} x_{n+\varepsilon}\right)$ and $\mathrm{E}\left(\left(x_{n} x_{n+\varepsilon}\right)^{*}\right)$ were omitted since in complex circular normal variables, these terms are zeroed, if the imaginary and real parts have the same variances. The same result is extracted for the remaining term of (69). The combined result is given by

$$
\mathrm{E}\left(\mathbf{y}_{i}^{\text {oqam }}\left(\mathbf{y}_{i}^{\text {oqam }}\right)^{\mathrm{H}}\right)=\sigma_{x, i}^{2} \mathbf{I}_{N}
$$

Similarly to the previous analysis, the correlation of the output from non-directly adjacent filter paths is considered zero. The next step is to calculate the crosscorrelation between outputs of directly adjacent filters. Assuming that filter path $i$ is even, then $i+1$ is odd. The exact same procedure can be used for an odd $i$ th filter. Thus, where once more, all the terms that did not contain products between conjugates were ignored. The first finding concerns the first four terms. Since the output from each separate filter bank is the same with the output of a uniform DFT filter bank at Nyquist rate, then the cross-correlation matrix between the outputs of adjacent paths coming from the same filter bank has already been calculated in (22) for white Gaussian input with unitary variance $(\mathcal{N}(0,1))$. Given that the only difference between filter banks (A) and (B) is a delay offset of the input and that filter paths $\mathbf{y}_{i}^{(\mathrm{A})}, \mathbf{y}_{i}^{(\mathrm{B})}$ monitor the same spectral band (with the same radio channel coefficient $c_{i}$ ), the output cross-correlations for adjacent subchannels for the two filter banks are equal. Therefore,

$$
\mathrm{E}\left(\mathbf{y}_{i}^{(\mathrm{A})}\left(\mathbf{y}_{i+1}^{(\mathrm{A})}\right)^{\mathrm{H}}\right)=\mathrm{E}\left(\mathbf{y}_{i}^{(\mathrm{B})}\left(\mathbf{y}_{i+1}^{(\mathrm{B})}\right)^{\mathrm{H}}\right)=\sigma_{x, i} \sigma_{x, i+1} \mathbf{R},
$$

$$
\begin{aligned}
& \mathrm{E}\left(\mathbf{y}_{i}^{\text {oqam }}\left(\mathbf{y}_{i+1}^{\text {oqam }}\right)^{\mathrm{H}}\right)=\mathrm{E}\left(\left(\frac{\mathbf{y}_{i}^{(\mathrm{A})}+\left(\mathbf{y}_{i}^{(\mathrm{A})}\right)^{*}}{2}+\frac{\mathbf{y}_{i}^{(\mathrm{B})}-\left(\mathbf{y}_{i}^{(\mathrm{B})}\right)^{*}}{2}\right)\left(\left(\frac{\mathbf{y}_{i+1}^{(\mathrm{A})}-\left(\mathbf{y}_{i+1}^{(\mathrm{A})}\right)^{*}}{2}\right)^{\mathrm{H}}+\left(\frac{\mathbf{y}_{i+1}^{(\mathrm{B})}+\left(\mathbf{y}_{i+1}^{(\mathrm{B})}\right)^{*}}{2}\right)^{\mathrm{H}}\right)\right. \\
& =\frac{1}{4}\left(\begin{array}{l}
\mathrm{E}\left(\mathbf{y}_{i}^{(\mathrm{A})}\left(\mathbf{y}_{i+1}^{(\mathrm{A})}\right)^{\mathrm{H}}\right)-\mathrm{E}\left(\left(\mathbf{y}_{i}^{(\mathrm{A})}\right)^{*}\left(\mathbf{y}_{i+1}^{(\mathrm{A})}\right)^{\mathrm{T}}\right)+\mathrm{E}\left(\mathbf{y}_{i}^{(\mathrm{B})}\left(\mathbf{y}_{i+1}^{(\mathrm{B})}\right)^{\mathrm{H}}\right)-\mathrm{E}\left(\left(\mathbf{y}_{i}^{(\mathrm{B})}\right)^{*}\left(\mathbf{y}_{i+1}^{(\mathrm{B})}\right)^{\mathrm{T}}\right) \\
+\mathrm{E}\left(\mathbf{y}_{i}^{(\mathrm{B})}\left(\mathbf{y}_{i+1}^{(\mathrm{A})}\right)^{\mathrm{H}}\right)+\mathrm{E}\left(\mathbf{y}_{i}^{(\mathrm{A})}\left(\mathbf{y}_{i+1}^{(\mathrm{B})}\right)^{\mathrm{H}}\right)+\mathrm{E}\left(\left(\mathbf{y}_{i}^{(\mathrm{B})}\right)^{*}\left(\mathbf{y}_{i+1}^{(\mathrm{A})}\right)^{\mathrm{T}}\right)+\mathrm{E}\left(\left(\mathbf{y}_{i}^{(\mathrm{A})}\right)^{*}\left(\mathbf{y}_{i+1}^{(\mathrm{B})}\right)^{\mathrm{T}}\right)
\end{array}\right)
\end{aligned}
$$


and given that $\mathbf{R}$ is real and symmetric:

$$
\begin{aligned}
\mathrm{E}\left(\left(\mathbf{y}_{i}^{(\mathrm{A})}\right)^{*}\left(\mathbf{y}_{i+1}^{(\mathrm{A})}\right)^{\mathrm{T}}\right) & =\mathrm{E}\left(\left(\mathbf{y}_{i}^{(\mathrm{B})}\right)^{*}\left(\mathbf{y}_{i+1}^{(\mathrm{B})}\right)^{\mathrm{T}}\right) \\
& =\sigma_{x, i} \sigma_{x, i+1} \mathbf{R}^{\mathrm{H}}=\sigma_{x, i} \sigma_{x, i+1} \mathbf{R}
\end{aligned}
$$

where $\sigma_{x, i}=\sigma_{s}\left|c_{i}\right|$. Therefore, the sum of the first four terms in (73) is zero. Regarding the following four terms, it can be proved after some mathematical manipulation that

$$
\begin{aligned}
{\left[\mathrm{E}\left(\mathbf{y}_{i}^{(\mathrm{A})}\left(\mathbf{y}_{i+l}^{(\mathrm{B})}\right)^{\mathrm{H}}\right)\right]_{k, m}=} & \sigma_{x, i} \sigma_{x, i+l}(-1)^{i+l} \\
& \times \sum_{u=-\infty}^{\infty} h_{u} h_{u-\frac{M}{2}+M \varepsilon} e^{-2 \pi j \frac{l u}{M}} \\
= & \sigma_{x, i} \sigma_{x, i+l}(-1)^{i+l}(-1)\left\lfloor 1+\frac{l}{2}\right\rfloor_{j} \\
& \times \sum_{u=-\infty}^{\infty} h_{u} h_{u-\frac{M}{2}+M \varepsilon} \sin \left(\frac{2 \pi u}{M}\right) \\
\triangleq & \sigma_{x, i} \sigma_{x, i+l}(-1)^{i+l}(-1)\left\lfloor 1+\frac{l}{2}\right\rfloor j g_{M \varepsilon-\frac{M}{2}}^{\sin }
\end{aligned}
$$

This result is achieved using the symmetry of the prototype FIR filter. Matrix $\mathbf{T}$ is defined in order to store the outcome for $\sigma_{x, i}=1, \sigma_{x, i+l}=1$. With unification of the sign manipulation factors, the matrix elements are given by

$$
\left[\mathbf{T}_{i, l}\right]_{k, m}=\left\{\begin{array}{l}
j(-1)^{i+1} g_{M(m-k)-\frac{M}{2}}^{\sin }, l=1 \\
j(-1)^{i} g_{M(m-k)-\frac{M}{2}}^{\sin }, l=-1
\end{array}\right.
$$

Based on (76) and given the fact that $\mathbf{T}$ is purely imaginary, it is also concluded that

$$
\begin{aligned}
{\left[\mathrm{E}\left(\left(\mathbf{y}_{i}^{(\mathrm{A})}\right)^{*}\left(\mathbf{y}_{i+l}^{(\mathrm{B})}\right)^{\mathrm{T}}\right)\right]_{k, m} } & =\left(\sigma_{x, i} \sigma_{x, i+l}\left[\mathbf{T}_{i, l}\right]_{k, m}\right)^{*} \\
& =-\sigma_{x, i} \sigma_{x, i+l}\left[\mathbf{T}_{i, l}\right]_{k, m} \\
\mathrm{E}\left(\mathbf{y}_{i+l}^{(\mathrm{B})}\left(\mathbf{y}_{i}^{(\mathrm{A})}\right)^{\mathrm{H}}\right) & =\sigma_{x, i} \sigma_{x, i+l} \mathbf{T}_{i, l}^{\mathrm{H}}, \mathrm{E}\left(\left(\mathbf{y}_{i+l}^{(\mathrm{B})}\right)^{*}\left(\mathbf{y}_{i}^{(\mathrm{A})}\right)^{\mathrm{T}}\right) \\
& =-\sigma_{x, i} \sigma_{x, i+l} \mathbf{T}_{i, l}^{\mathrm{H}}
\end{aligned}
$$

Combining (77) and (74) in (73), the result is given by

$$
\mathrm{E}\left(\mathbf{y}_{i}^{\text {oqam }}\left(\mathbf{y}_{i+1}^{\text {oqam }}\right)^{\mathrm{H}}\right)=\mathbf{0}_{N}
$$

Thus, if the MDFT filter bank output after the operators $\Re(\bullet)$ and $\Im(\bullet)$ is used, then all the signal vectors that participate in the detection procedure are uncorrelated. The observation vector is defined as

$$
\mathbf{y}^{\text {oqam }}=\left[\left(\mathbf{y}_{0}^{\text {oqam }}\right)^{\mathrm{T}}\left(\mathbf{y}_{1}^{\text {oqam }}\right)^{\mathrm{T}} \ldots\left(\mathbf{y}_{1}^{\text {oqam }}\right)^{\mathrm{T}}\right]^{\mathrm{T}}
$$

where due to the independence of signals, reordering is not needed. The covariance matrix of $y^{\text {oqam }}$ is the following $L N \times L N$ matrix:

$$
\mathrm{E}\left(\mathbf{y}^{\text {oqam }}\left(\mathbf{y}^{\text {oqam }}\right)^{\mathrm{H}}\right)=\left[\begin{array}{cccc}
\sigma_{x, 0}^{2} \mathbf{I}_{N} & \mathbf{0}_{N} & \ldots & \mathbf{0}_{N} \\
\mathbf{0}_{N} & \sigma_{x, 1}^{2} \mathbf{I}_{N} & & \vdots \\
\vdots & & \ddots & \mathbf{0}_{N} \\
\mathbf{0}_{N} & \ldots & \mathbf{0}_{N} & \sigma_{x, L-1}^{2} \mathbf{I}_{N}
\end{array}\right]
$$

The form of this matrix leads to the pleasant conclusion that the weighted energy detector (with proper scaling for each filter path) is the Neyman-Pearson optimum detector. The metric of the detector is given by

$$
T_{\text {oqam }}(\mathbf{y})=\frac{\sigma_{s}^{2}}{\sigma_{w}^{2}} \sum_{i=0}^{L-1} \frac{\left|c_{i}\right|^{2}}{\sigma_{s}^{2}\left|c_{i}\right|^{2}+\sigma_{w}^{2}}\left(\mathbf{y}_{i}^{(\text {oqam })}\right)^{\mathrm{H}} \mathbf{y}_{i}^{(\mathrm{oqam})}
$$

The metric distribution can be extracted by

- With the use of the analytical method of (55), (56) and (58) using as eigenvalues the detector weights, i.e.

$$
\begin{aligned}
\lambda_{i}^{\mathrm{oqam} / \mathcal{H}_{0}} & =\frac{\sigma_{s}^{2}\left|c_{i}\right|^{2}}{\left(\sigma_{w}^{2}+\sigma_{s}^{2}\left|c_{i}\right|^{2}\right)} \\
\lambda_{i}^{\mathrm{oqam} / \mathcal{H}_{1}} & =\frac{\sigma_{s}^{2}\left|c_{i}\right|^{2}}{\sigma_{w}^{2}}
\end{aligned}
$$

- Or using the FFT-based numerical integration with $\varphi_{T\left(\mathbf{y}_{\text {oqam }} / H_{m}\right)}(\omega)=\prod_{i=0}^{L-1}\left(1-j \lambda_{i}^{\text {oqam } / \mathcal{H}_{m}} \omega\right)^{-N}$ for the outcomes $m=0,1$.

In Figure 5, the histogram of the simulated OFDMOQAM detector metric is compared with the theoretically calculated distribution. Both methods of distribution calculation return practically identical results. A very similar figure can be extracted for the NeymanPearson detector for uniform DFT filter banks, since both optimal detectors have identical metric distributions. The results of the two optimum Neyman-Pearson detectors for the uniform DFT and the MDFT filter bank present similar performance since in both cases the maximum possible amount of information is used. The MDFT filter bank uses double computational workload for filtering. However, in most cases for relatively large sample sets and $L>3$, the matrix multiplications for the computation of the uniform DFT metric lead to heavier computational burden. Therefore, in general, the OFDM-OQAM optimum detection is favourable. 


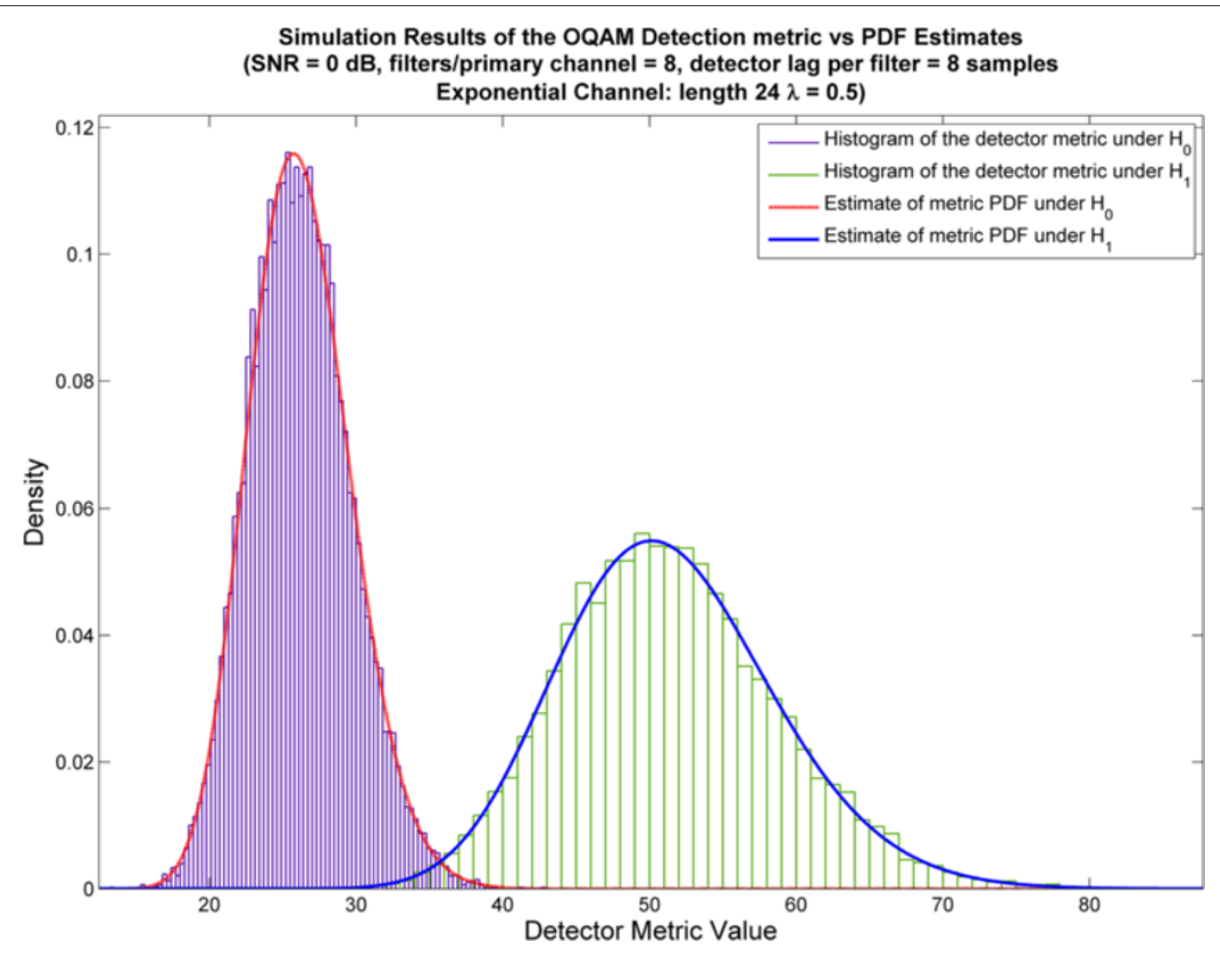

Figure 5 Distribution fit example for the Neyman-Pearson detection metric for an MDFT filter bank.

\section{Energy detection in the uniform DFT filter bank}

The optimum detector for an MDFT filter bank in Section 4 is proven to be a weighted (per subchannel) energy detector. Although the performance is identical, the uniform DFT optimum detector is much more complicated since it involves multiplications with the matrix $\mathbf{C}^{-1}$. In this section, the use of the weighted energy detector in a uniform DFT is investigated. It can be proved that the weighted energy detector is optimal if $\mathbf{R}=\mathbf{0}$; however, this is not possible in a DFT filter bank with a root-Nyquist prototype filter.

The weighted energy detector as a filter bank-based algorithm was presented in [26], where it was assumed that the subchannel filter outputs are uncorrelated and that the metric performance is determined using Gaussian distributions. It is also claimed that the weighted energy detector operation with optimal weights is similar to the maximum ratio combining (MRC) for independent variables; however, the optimal weights are not determined. The MRC consideration is proved to be the correct and optimum approach for the OFDM-OQAM detector in Section 4, where filter outputs are uncorrelated. In this section, an extension and generalization of the weighted energy detector is performed. The subchannel filter outputs are considered correlated, a fact that is inevitable for uniform DFT filter banks. Moreover, accurate and analytic distributions for the metric under $\mathcal{H}_{0}$ and $\mathcal{H}_{1}$ are extracted based on probability theory.
The metric of the weighted energy detector is given by

$$
T_{e d}(\mathbf{y})=\frac{\sigma_{s}^{2}}{\sigma_{w}^{2}} \sum_{i=0}^{L-1} \frac{\left|c_{i}\right|^{2}}{\sigma_{s}^{2}\left|c_{i}\right|^{2}+\sigma_{w}^{2}} \mathbf{y}_{i}^{\mathrm{H}} \mathbf{y}_{i}=\sum_{i=0}^{L-1} T_{i}\left(\mathbf{y}_{i}\right)
$$

For zero-mean complex Gaussian input, additive white noise and root-Nyquist prototype filter, the output for each subchannel is proved to follow the distributions below:

$$
\begin{aligned}
& \mathcal{H}_{0}: T_{i}\left(\mathbf{y}_{i}\right) \sim \mathcal{G}\left(N, \frac{\sigma_{s}^{2}\left|c_{i}\right|^{2}}{\sigma_{s}^{2}\left|c_{i}\right|^{2}+\sigma_{w}^{2}}\right) \\
& \mathcal{H}_{1}: T_{i}\left(\mathbf{y}_{i}\right) \sim \mathcal{G}\left(N, \frac{\sigma_{s}^{2}\left|c_{i}\right|^{2}}{\sigma_{w}^{2}}\right)
\end{aligned}
$$

Since independence between samples from the adjacent filters $\mathbf{y}_{i+1}$ and $\mathbf{y}_{i-1}$ is not possible, the performance of the detector will be inferior to the detector in Section 3 . The hysteresis of the detector depends on the transition bandwidth and the roll-off of the prototype filter.

Therefore, $T_{\text {ed }}(\mathbf{y})$ is a sum of weighted, correlated gamma variables. In order to extract the metric distribution, it is necessary to calculate the correlation coefficient for the energy between the outputs of two adjacent filter paths. According to [37] (Jensen approximation), it is claimed that for a stream of $N$ pairs of samples of Gaussian variables $z_{k}=\left(z_{k 1}, z_{k 2}\right)$ with $(\mu=0, \sigma=1)$ that are mutually correlated (but each one has uncorrelated time samples), then the correlation of the energies $u_{1}=$ 


$$
\begin{gathered}
\sum_{k=0}^{N-1}\left|z_{k 1}^{2}\right|, u_{2}=\sum_{k=0}^{N-1}\left|z_{k 2}^{2}\right| \text { can be calculated by } \\
p_{u_{1} u_{2}}=\frac{1}{N} \sum_{n=0}^{N-1} q_{n}^{2}
\end{gathered}
$$

where the $q_{n}$ s are the normal correlations for variables $\left(z_{k 1}, z_{k 2}\right)$. Jensen's result is valid for real random variables with standard normal distribution. However, it can easily be proved through the correlation coefficient that the same formula is valid for $2 N$-length real normal variables with $\mu=0, \sigma^{2}=1 / 2$. Since a $N$-complex normal vector is equivalent with a $2 N$-real vector, the Jensen's corollary can be used for the current analysis.

Normal correlations are the means to quantify the linear relationship between two multivariate random variables. The normal correlations can be calculated if the joint covariance matrix of the two random variables is known. Specifically, for known covariance matrix,

$$
\left[\begin{array}{ll}
\mathbf{C}_{\mathbf{x}_{1} \mathbf{x}_{1}} & \mathbf{C}_{\mathbf{x}_{1} \mathbf{x}_{2}} \\
\mathbf{C}_{\mathbf{x}_{2} \mathbf{x}_{1}} & \mathbf{C}_{\mathbf{x}_{2} \mathbf{x}_{2}}
\end{array}\right] \text {, for the variables } \mathbf{x}_{1}, \mathbf{x}_{2}
$$

the normal correlation squares are given as the eigenvalues of the matrix:

$$
\begin{aligned}
& \mathbf{C}_{\mathbf{x}_{1} \mathbf{x}_{1}}^{-1} \mathbf{C}_{\mathbf{x}_{1} \mathbf{x}_{2}} \mathbf{C}_{\mathbf{x}_{2} \mathbf{x}_{2}}^{-1} \mathbf{C}_{\mathbf{x}_{2} \mathbf{x}_{1} \mathbf{w}_{\mathbf{x}}}=q_{\mathbf{x}_{1} \mathbf{x}_{2}}^{2} \mathbf{w}_{\mathbf{x}} \\
& \mathbf{C}_{\mathbf{x}_{2} \mathbf{x}_{2}}^{-1} \mathbf{C}_{\mathbf{x}_{2} \mathbf{x}_{1}} \mathbf{C}_{\mathbf{x}_{1} \mathbf{x}_{1}}^{-1} \mathbf{C}_{\mathbf{x}_{1} \mathbf{x}_{2} \mathbf{w}_{\mathbf{y}}}=q_{\mathbf{x}_{1} \mathbf{x}_{2}}^{2} \mathbf{w}_{\mathbf{y}}
\end{aligned}
$$

where $\mathbf{w}_{\mathbf{x}, \mathbf{y}}$ are the respective eigenvectors. The problem is to calculate the correlation coefficient between the energies of $N$ output samples from adjacent filter banks. The covariance matrix $\mathbf{y}_{i}, \mathbf{y}_{i+1}$ is given by (Section 3 ):

$E\left(\left[\begin{array}{ll}\mathbf{y}_{i}^{\mathrm{H}} & \mathbf{y}_{i+1}^{\mathrm{H}}\end{array}\right]\left[\begin{array}{c}\mathbf{y}_{i} \\ \mathbf{y}_{i+1}\end{array}\right]\right)=\sigma_{s}^{2}\left[\begin{array}{cc}\left|c_{i}\right|^{2} \mathbf{I}_{N} & \left|c_{i}\right|\left|c_{i+1}\right| \mathbf{R} \\ \left|c_{i}\right|\left|c_{i+1}\right| \mathbf{R}^{\mathrm{H}} & \left|c_{i+1}\right|^{2} \mathbf{I}_{N}\end{array}\right]$

Given that $\mathbf{R}$ is hermitian, it can be proved from (87) that the vector $\mathbf{q}_{\mathbf{y}_{i} \mathbf{y}_{i+1}}^{2}$ is also the vector of eigenvalues of matrix $\mathbf{R}^{2}$. The correlation coefficient can be extracted as the sum of the squares of normal correlations. Since the matrix trace $(\mathrm{Tr})$ is equal to the sum of the eigenvalues, it is proved that

$$
\operatorname{Tr}\left[\mathbf{R}^{2}\right]=\sum_{n=0}^{N-1} q_{\mathbf{y}_{i} \mathbf{y}_{i+1}, n}^{2}
$$

The correlation coefficient is equal to the mean of the $\mathbf{R}^{2}$ eigenvalues, i.e. $p_{\mathbf{y}_{i} \mathbf{y}_{i+1}}=\operatorname{Tr}\left[\mathbf{R}^{2}\right] / N$

Another important note is that since the correlation coefficient does not depend on the radio channel coefficients, it is the same for every pair of adjacent subchannel outputs. It is also noted that for non-directly adjacent subchannels, the correlation coefficient is assumed to be zero. Once again, the analysis in [36] can be followed in order to extract the metric distribution. According to [36], if $\left\{u_{i}\right\}_{i=1}^{L-1}$ is a set of $L$-correlated gamma variables with equal degrees of freedom and known correlation coefficients, then the PDF can be expressed analytically. Unlike the results in Sections 3 and 4 where an identity correlation matrix is considered, in this case, the positive definite matrix $\mathbf{C}_{\text {ed }}$ that expresses the correlations is given by [36]

$$
\mathbf{C}_{\mathrm{ed}}=\left[\begin{array}{ccccc}
1 & \sqrt{p} & 0 & \ldots & 0 \\
\sqrt{p} & 1 & \sqrt{p} & & \vdots \\
0 & \sqrt{p} & 1 & \ddots & 0 \\
\vdots & & \ddots & \ddots & \sqrt{p} \\
0 & \ldots & 0 & \sqrt{p} & 1
\end{array}\right]
$$

The tridiagonal matrix is an $L \times L$ matrix. As a next step, the diagonal matrix $\mathbf{D}_{\mathrm{ed}}$ is defined according to the rule:

$$
\begin{aligned}
& \mathbf{D}_{\text {ed }}=\operatorname{diag}\left(\left[\frac{\sigma_{s}^{2}\left|c_{0}\right|^{2}}{\sigma_{s}^{2}\left|c_{0}\right|^{2}+\sigma_{w}^{2}} \cdots \frac{\sigma_{s}^{2}\left|c_{L-1}\right|^{2}}{\sigma_{s}^{2}\left|c_{L-1}\right|^{2}+\sigma_{w}^{2}}\right]\right), \text { for } \mathcal{H}_{0} \\
& \mathbf{D}_{\text {ed }}=\operatorname{diag}\left(\left[\frac{\sigma_{s}^{2}\left|c_{0}\right|^{2}}{\sigma_{w}^{2}} \cdots \frac{\sigma_{s}^{2}\left|c_{L-1}\right|^{2}}{\sigma_{w}^{w}}\right]\right), \text { for } \mathcal{H}_{1}
\end{aligned}
$$

The distributions are calculated by (55), (56), (58) and (66) using the eigenvalues $\lambda_{i}^{\text {ed/ } / \mathcal{H}_{0}}$ and $\lambda_{i}^{\text {ed } / \mathcal{H}_{1}}$ that are calculated from the matrix: $\mathbf{A}_{\mathrm{ed}}=\mathbf{D}_{\mathrm{ed}} \mathbf{C}_{\mathrm{ed}}$. Since $\mathbf{A}_{\mathrm{ed}}$ is tridiagonal, the eigenvalues can be calculated iteratively [38]. A distribution fit example for the weighted energy detector metric is presented in Figure 6.

In [26], it is mentioned that for uncorrelated, independent subchannel filter outputs, the weighted energy detector can be seen as an approximation of the matched filtering. This is accurate for the OFDM-OQAM NeymanPearson (NP) detector where subchannel independence is proved. More specifically, if the 'approximately flat radio channel per subchannel' assumption is valid and the radio channel can be adequately described by the $c_{i}$ parameters, then the matched filter approximation is exceptional. As the channel frequency selectivity increases, the deviation from the matched filter performance also increases. However, in the common case of correlated filter outputs, the deviation from the matched filter performance is significant. Since the weighted energy detector metric ignores the correlation of the output samples, it fails to perfectly align the decision metrics in order to maximize probability of detection. This is achieved by the NP optimal detector of Section 3. In terms of diversity, the weighted energy detector is transformed into an non-optimal MRC that ignores the existed correlation among the diversity branches. Despite the fact that the weighted energy detector is outperformed by the NP detector, the selection of weights that depends on the SNR per subchannel provides significant advantages over the conventional energy detector. The integration of the radio channel effects in the detector with the use of the 


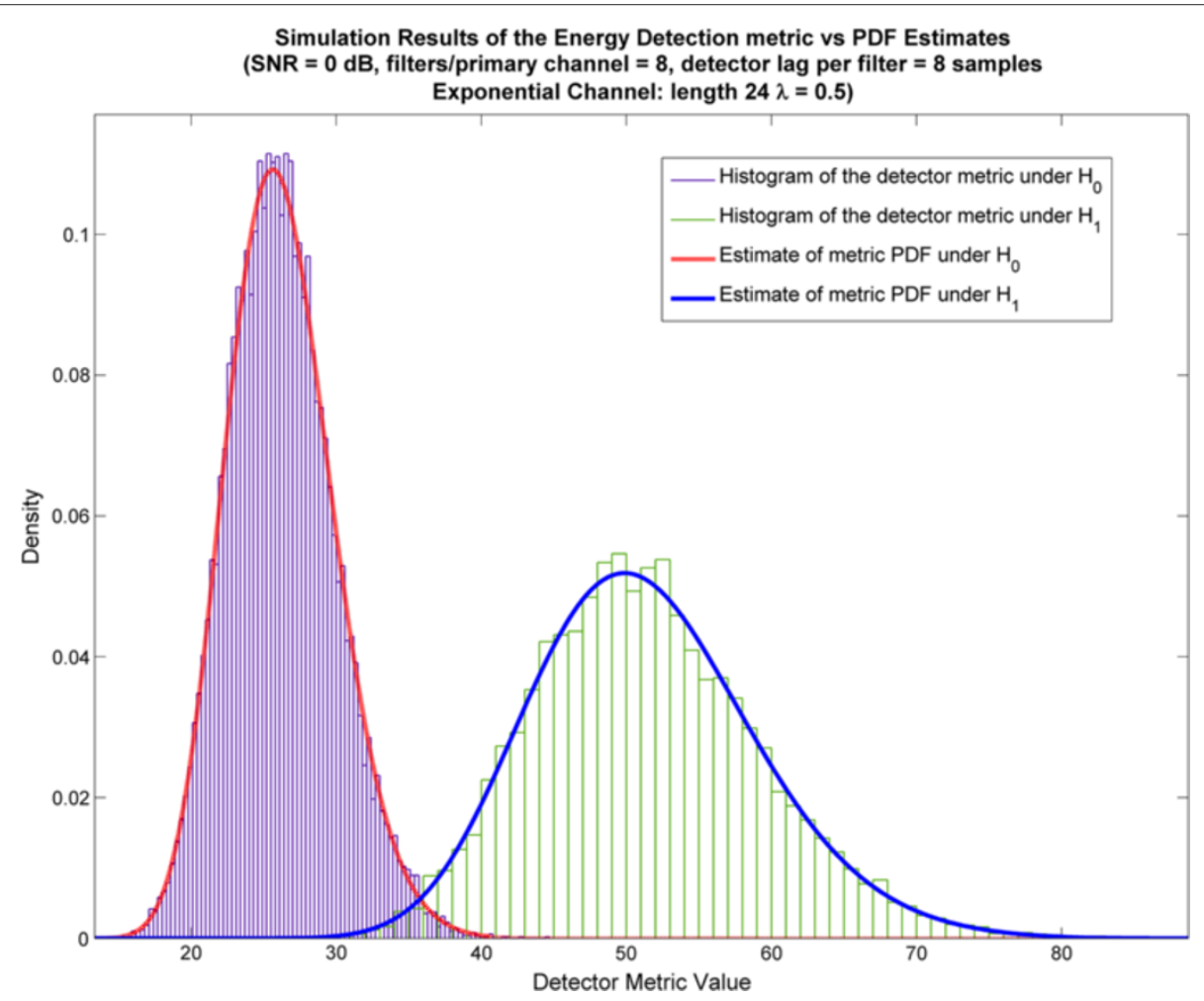

Figure 6 Distribution fit example for the weighted energy detector matrix for uniform DFT filter bank.

$c_{i}$ parameters is essential, despite the fact that it is based on the 'approximately flat radio channel' approximation. Thus, the performance degradation of the weighted energy detector from the NP optimal detector is much smaller than the achieved improvement from the performance of the conventional energy detector, as presented in Figure 7.

\section{Coloured noise}

An important weakness of many detectors is that they are not robust in the existence of coloured noise. Coloured noise may be the result of interference caused by remote PSs or CRs, it may be caused by various electronic noise sources or it may appear due to filter leakage and spurious emissions of users operating in adjacent spectrum bands [5]. In this section, additive coloured Gaussian noise is assumed. In AWGN models, the noise samples are uncorrelated. Coloured noise can be on the other hand modelled as the result of filtering AWGN samples. When the noise reaches the receiver, it is subjected also to filtering from the filter bank. Similarly to the assumption regarding the primary signal, the noise variance is considered approximately constant for the bandpass zone of each filter path. Therefore, the coloured noise modelling can be performed with the following relationships:

$$
\begin{aligned}
& S_{c w}(f)=S_{w w}(f)|V(f)|^{2}\left|H\left(f-f_{i}\right)\right|^{2} \Rightarrow \\
& S_{c w}(f) \simeq \sigma_{w}^{2}|V(f)|^{2}\left|H\left(f-f_{i}\right)\right|^{2}
\end{aligned}
$$

where $S_{c w}$ is the PSD of the coloured noise, $S_{w w}$ is the AWGN PSD, $V(f)$ is the transfer function that colours the noise, $H(f)$ is the prototype filter transfer function for the filter bank. Similarly to the assumption for frequency selective channels expressed in (11), it can be assumed that the additive noise at the output of each filter of the bank is also white with variance given by

$$
\sigma_{w, i}^{2}=\frac{\sigma_{w}^{2}\left|V\left(f_{i}\right)\right|}{\mathrm{BW}} \int_{-\infty}^{\infty}\left|H\left(f-f_{i}\right)\right|^{2} d f
$$

where BW is the overall bandwidth.

Coloured noise may be the result of the following: (a) out-of-band emissions and adjacent channel interference, (b) aliasing effects and (c) non-uniform distribution of interferences from remote cells and access points. Filter bank-based sensing can robustly detect signals under coloured noise. The diagonal matrix $\boldsymbol{\Sigma}_{w}$ can 
be defined that contains the (assumed constant) noise variance per subchannel. For the uniform DFT NeymanPearson detector, the matrix $\boldsymbol{\Sigma}_{w}$ is defined as

$$
\begin{aligned}
\boldsymbol{\Sigma}_{w} & =\left[\begin{array}{cc}
\boldsymbol{\Sigma}_{w}^{(0)} & \mathbf{0} \\
\mathbf{0} & \boldsymbol{\Sigma}_{w}^{(1)}
\end{array}\right], \text { where } \\
\boldsymbol{\Sigma}_{w}^{(0)} & =\operatorname{diag}\left[\begin{array}{llll}
\sigma_{w, 0} \mathbf{I}_{N} & \sigma_{w, 2} \mathbf{I}_{N} & \ldots & \sigma_{w, L-2} \mathbf{I}_{N}
\end{array}\right] \\
\boldsymbol{\Sigma}_{w}^{(1)} & =\operatorname{diag}\left[\begin{array}{llll}
\sigma_{w, 1} \mathbf{I}_{N} & \sigma_{w, 3} \mathbf{I}_{N} & \ldots & \sigma_{w, L-1} \mathbf{I}_{N}
\end{array}\right]
\end{aligned}
$$

Under these circumstances, the standard deviation matrix $\boldsymbol{\Sigma}_{y}$ for the incoming signal under $\mathcal{H}_{1}$ is given by $\boldsymbol{\Sigma}_{y}=$ $\left(\Sigma^{2}+\Sigma_{w}^{2}\right)^{1 / 2}$. The detection metric can be written as

$$
\begin{aligned}
T(\mathbf{y}) & =\mathbf{y}^{\mathrm{H}}\left(\mathbf{C}_{w}^{-1}-\mathbf{C}_{y}^{-1}\right) \mathbf{y}= \\
& =\mathbf{y}^{\mathrm{H}} \Sigma_{w}^{-1}\left(\mathbf{C}^{-1}+\Sigma_{w} \Sigma_{y}^{-1} \mathbf{C}^{-1} \Sigma_{y}^{-1} \Sigma_{w}\right) \Sigma_{w}^{-1} \mathbf{y}
\end{aligned}
$$

The result in (95) shows that with simple weighting of the observation samples by $\mathbf{y}^{\prime}=\boldsymbol{\Sigma}_{w}^{-1} \mathbf{y}$, the problem is transformed to the equivalent Neyman-Pearson detector with
AWGN. More specifically, the equivalent AWGN problem is defined with $\sigma_{w}^{2}=1$ and $\left|c^{\prime}{ }_{i}\right|=\frac{\left|c^{\prime} i\right|}{\sigma_{w, i}}$.

\section{Practical implementation of the detectors}

\subsection{The locally most powerful test}

The main disadvantage of the sensing techniques described in the previous sections is that the detector should know the signal variance for each subchannel (the received signal power and the channel coefficient). In real-world implementations, the optimal detectors can be approximated with the use of estimates; however, these detectors are only optimal asymptotically. In case the information for the signal variance is missing or primarily when the subchannel variances are extremely small (linearly expressed SNR $\rightarrow$ 0), then the locally most powerful test can be used for detection. A composite detection problem is considered to have a uniformly LMP detector, when both the metric and the threshold for a given $P_{\text {FA }}$ do not depend on unknown parameters (in this case the variances). Unfortunately, these cases are very rare.

In this section, the LMP detector is formed for a uniform DFT filter bank. The observation vector is defined as in (5) but the binary problem is now defined as follows:

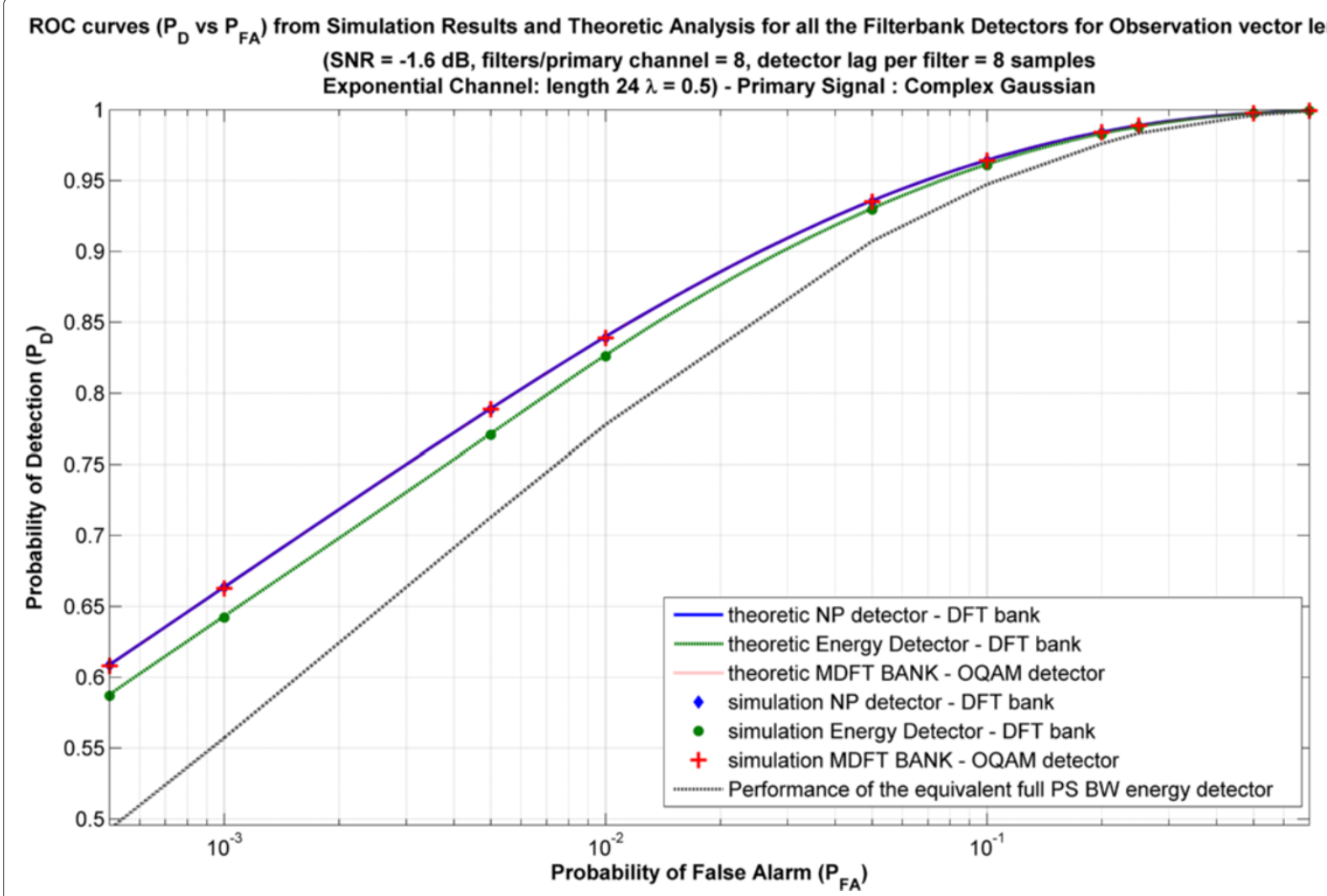

Figure 7 ROC curve $P_{D}$ vs. $P_{F A}$ for detection under AWGN. 
For: $\mathcal{H}_{0}$ :

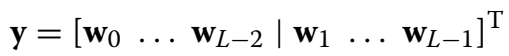

$$
\begin{aligned}
& \text { For: } \mathcal{H}_{1} \text { : }
\end{aligned}
$$

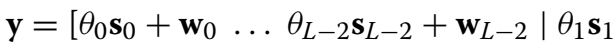

$$
\begin{aligned}
& \left.+\mathbf{w}_{1} \ldots \theta_{L-1} \mathbf{s}_{L-1}+\mathbf{w}_{L-1}\right]^{\mathrm{T}} \\
& \left(\boldsymbol{\theta}=\left[\begin{array}{llllll}
\theta_{0} & \ldots & \theta_{L-2} & \theta_{1} & \ldots & \theta_{L-1}
\end{array}\right)\right.
\end{aligned}
$$

The noise is defined as in the previously described detectors. On the other hand, the signals $\mathbf{s}_{i}$ are considered standard complex normal variables. Each signal is multiplied with a $\theta_{i}$ parameter close to zero. Due to the filtering, the outputs of the adjacent subchannels are correlated through the matrix $\mathbf{C}(24)$. The binary problem is formulated as

$$
\begin{aligned}
& \mathcal{H}_{0}: \boldsymbol{\theta}=\mathbf{0}_{L, 1} \\
& \mathcal{H}_{1}: \boldsymbol{\theta}>\mathbf{0}_{L, 1}
\end{aligned}
$$

where the vector inequality has the following meaning: If there is a primary signal in the specific subchannels, it should be $\theta_{i}>0$ for all $i=0,1 \ldots L-1$. Using the procedure defined in $[24,39]$, it is found that since the vector $\boldsymbol{\theta}$ contains a set of $L$ parameters, the extraction of the LMP test becomes extremely complicated. This problem can be bypassed using a simple consideration. Since $\boldsymbol{\theta}$ values are extremely small (near to zero), they can be assumed as approximately equal and therefore, a scalar parameter $\theta$ common for all the subchannel outputs can be used. This is also verified by the fact that the LMP tests for simple structures (e.g. two filters, one sample per filter for the decision) are identical either if $L$ parameters or a single parameter is assumed. Thus, the binary problem is simplified:

$$
\begin{aligned}
& \mathcal{H}_{0}: \theta=0 \\
& \mathcal{H}_{1}: \theta>0
\end{aligned}
$$

According to Section 3 and using the common scalar parameter $\theta$, the observation vector distributions are given by

$$
\begin{aligned}
& \mathcal{H}_{0}: \mathbf{y} \sim \mathcal{N}\left(0, \mathbf{C}_{w}=\sigma_{w}^{2} \mathbf{C}\right) \\
& \mathcal{H}_{1}: \mathbf{y} \sim \mathcal{N}\left(0, \mathbf{C}_{y}=\mathbf{\Sigma} \mathbf{C} \boldsymbol{\Sigma}+\sigma_{w}^{2} \mathbf{C}=\left(\theta^{2}+\sigma_{w}^{2}\right) \mathbf{C}\right)
\end{aligned}
$$

Since $\theta \rightarrow 0$, the PDF can be approximated using the Taylor series for $\theta^{(0)}=0$ :

$$
\begin{aligned}
& \ln \left(f\left(\mathbf{y} ; \theta / \mathcal{H}_{1}\right)\right) \simeq \ln \left(f\left(\mathbf{y} ; \theta=0 / \mathcal{H}_{1}\right)\right)+ \\
& \left.\theta \frac{\partial\left(\ln \left(f\left(\mathbf{y} ; \theta / \mathcal{H}_{1}\right)\right)\right)}{\partial \theta}\right|_{\theta=0}+\left.\frac{\theta^{2}}{2} \frac{\partial^{2}\left(\ln \left(f\left(\mathbf{y} ; \theta / \mathcal{H}_{1}\right)\right)\right)}{\partial \theta^{2}}\right|_{\theta=0}
\end{aligned}
$$

where only the two first terms of the series are kept. It is proved using $\ln \left(f\left(\mathbf{y} ; \theta=0 / \mathcal{H}_{1}\right)\right)=\ln \left(f\left(\mathbf{y} / \mathcal{H}_{0}\right)\right)$ that

$$
\ln \left(f\left(\mathbf{y} ; \theta / \mathcal{H}_{1}\right)\right) \simeq \ln \left(f\left(\mathbf{y} / \mathcal{H}_{0}\right)\right)-\frac{N L}{\sigma_{w}^{2}}+\frac{1}{\sigma_{w}^{2}} \mathbf{y}^{\mathrm{H}} \mathbf{C}^{-1} \mathbf{y}
$$

Therefore, the detector decides using the following inequality:

$$
T_{\operatorname{lmp}}(\mathbf{y})=\mathbf{y}^{\mathrm{H}} \mathbf{C}^{-1} \mathbf{y} \underset{\mathcal{H}_{0}}{\stackrel{\mathcal{H}_{1}}{\gtrless}} v^{\prime}=\sigma_{w}^{2} \ln (v)+N L
$$

With the definition of the interim variable $\mathbf{z}$ as in (45), it can be proved that

$$
T_{\operatorname{lmp}}(\mathbf{y})=\sigma_{w}^{2} \mathbf{z}^{\mathrm{H}} \Lambda^{1 / 2} \mathbf{U}^{\mathrm{H}} \mathbf{C}^{-1} \mathbf{U} \Lambda^{1 / 2} \mathbf{z}=\sigma_{w}^{2} \mathbf{z}^{\mathrm{H}} \mathbf{z}
$$

which means that $t=T_{\operatorname{lmp}}\left(\mathbf{y} / \mathcal{H}_{0}\right) \sim \mathcal{G}\left(N L, \sigma_{w}^{2}\right)$ and the $P_{\text {FA }}$ is provided by

$$
P_{\mathrm{FA}}\left(v^{\prime}\right)=1-\frac{\gamma\left(N L, \frac{v^{\prime}}{\sigma_{w}^{2}}\right)}{\Gamma(N L)}
$$

Since $\theta$ remains unknown, the accurate calculation of $P_{\mathrm{D}}$ is not possible for the LMP test.

The prescribed procedure can be followed for the extraction of the LMP detector for MDFT filter banks. In this case, the covariance matrix is approximated by

$$
\mathbf{C}_{\text {oqam }}=\left(\sigma_{w}^{2}+\theta^{2}\right) \mathbf{I}_{N L}
$$

and the LMP metric is given by

$$
T_{\operatorname{lmp}}\left(\mathbf{y}_{\text {oqam }}\right)=\mathbf{y}_{\text {oqam }}^{H} \mathbf{y}_{\text {oqam }} \underset{\mathcal{H}_{0}}{\stackrel{\mathcal{H}_{1}}{\gtrless}} v^{\prime}=\sigma_{w}^{2} \ln (v)+N L
$$

which is the simple energy detector. The $P_{\text {FA }}$ can be calculated from (104) with $\sigma_{w}=1$. Therefore, the MDFT filter bank has a uniformly LMP test.

\subsection{The GLRT detector}

In a real-world implementation of the Neyman-Pearson detectors, the CR receiver is assumed to have no knowledge regarding the primary signal. In this section, an approximation of the optimal detectors using estimates is attempted. The receiver is assumed to know the noise power and the distribution family (zero mean complex Gaussian) for the primary signal under $\mathcal{H}_{1}$. This is a composite hypothesis test since the PDF under $\mathcal{H}_{1}$ depends on $L$ unknown parameters. The LMP, although it ensures constant $P_{\text {FA }}$, does not fully exploit the available information and it does not provide information or control over the achieved $P_{\mathrm{D}}$. Another approach is the Generalized Likelihood Ratio Test - GLRT. The GLRT decides using the log-likelihood ratio (as in the Neyman-Pearson 
detectors) and the maximum likelihood estimates (MLE) of the unknown distribution parameters.

$$
\begin{array}{r}
L_{G}(\mathbf{y})=\frac{f\left(\mathbf{y} / \widehat{\boldsymbol{\theta}}, \mathcal{H}_{1}\right)}{f\left(\mathbf{y} / \mathcal{H}_{0}\right)}, \text { where } \\
f\left(\mathbf{y} / \widehat{\boldsymbol{\theta}}, \mathcal{H}_{1}\right)=\max _{\widehat{\boldsymbol{\theta}}}\left(f\left(\mathbf{y} ; \widehat{\boldsymbol{\theta}} / \mathcal{H}_{1}\right)\right)
\end{array}
$$

where $\widehat{\boldsymbol{\theta}}$ is a vector containing the estimates of the unknown parameters under $\mathcal{H}_{1}$. The MLE is unbiased and asymptotically optimum and efficient. The basic definition of the GLRT defines that both estimation (of the parameters) and detection of the signal is based on the information from the observation vector. However, the results can be significantly improved using memory, i.e. previous observation vectors. The estimation procedure is assigned to each subchannel separately. The use of the joint $y$ distribution for estimation is avoided, since the estimation gain is very small compared to the increase of computational workload.

Per receiver subchannel, the signal is considered to be a white Gaussian variable, where the channel coefficient is replaced with parameter $\theta_{i}$. The MLE is proved to be equal to

$$
\hat{\theta}_{i}^{2}=\frac{\mathbf{y}_{i}^{\mathrm{H}} \mathbf{y}_{i}}{N}-\sigma_{w}^{2}
$$

The variance of the MLE $\hat{\theta}_{i}^{2}$ under $\mathcal{H}_{1}$ can be given by $\operatorname{var}\left[\hat{\theta}_{i}^{2}\right]=\frac{\left(\sigma_{w}^{2}+\theta_{i}^{2}\right)^{2}}{N}$, where $\theta_{i}^{2}$ is the exact value of the parameter. In this case, the MLE is the best possible estimate since it can be proved that it is equal with the Cramer-Rao bound. However, the estimator has a basic weakness. The estimate of (108) can take a negative value for a given measurement when $\theta_{i}^{2}$ tends to zero. For these cases, the estimate is considered unacceptable and it is rejected.
As mentioned before, the estimate can be improved with the use of previous measurements. Nevertheless, in spectrum sensing applications the specific tactic may bring instability. Let us consider the case of transition from $\mathcal{H}_{1}$ to $\mathcal{H}_{0}$. Thus, the previous observation vectors carry a primary signal (+ noise), but the current vector will contain only noise. The use of the current vector for parameter estimation will lead to higher estimation error. Therefore, the algorithm presented in Figure 8 is proposed.

Based on this algorithm, the initialization of the detector is made using the LMP test. When the LMP detects a primary signal, the GLRT detector is enabled. Each time a new detection is performed, the estimate from the previous measurements is used to built the detector. If the outcome of the new decision is also $\mathcal{H}_{1}$, then the estimate is updated using the current measurement. In order to update the estimate, a recursive MLE scheme can be used with a forgetting factor $\alpha \in\left[\begin{array}{ll}0 & 1\end{array}\right]$. More specifically, the updated estimate is given by

$$
\theta_{i}\left(t_{n+1}\right)=\alpha \theta_{i}\left(t_{n}\right)+(1-\alpha)\left(\frac{\mathbf{y}_{i}^{\mathrm{H}} \mathbf{y}_{i}}{N}-\sigma_{w}^{2}\right)^{+}
$$

where the superscript $(+)$ indicates that the current estimate is used only if positive; otherwise, it is set to zero. The forgetting factor also allows the detector to adjust in power variations of the incoming signal due to the time-variant nature of the radio channel.

In modern wireless systems, the use of time division multiple access, time duplexing and the bursty mode of data transmission indicate that a transceiver may perform interrupted transmissions. Thus, if $\mathcal{H}_{0}$ is decided, then the previous estimate should be kept in order for the GLRT to operate when/if the primary signal reappears. On the other hand, if a different primary user occupy the channel, then the outcomes are the following:

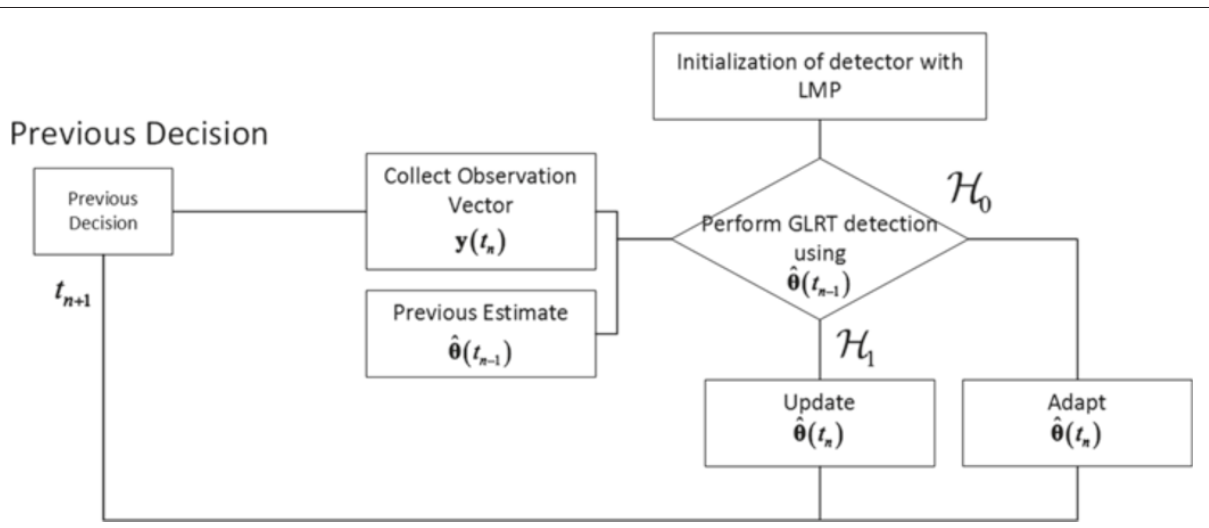

Figure 8 A block diagram describing an implementable design of the GLRT detector. 
- If the new signal is more powerful, then the GLRT (with the old signal estimate) will most probably decide on $\mathcal{H}_{1}$.

- If the new signal is less powerful, it becomes more probable to have a missed detection. In order to control this effect, an empirical scheme can be defined. It is assumed that the estimate of the signal power is partially reduced every time the outcome $\mathcal{H}_{0}$ is repeated. A rule that can be used for example is

$$
\theta_{i}\left(t_{n+1}\right)=\beta^{(n-\delta)^{+}} \theta_{i}\left(t_{n}\right)
$$

with $\beta \in\left[\begin{array}{ll}0 & 1\end{array}\right]$ a power reduction factor and $\delta$ a coefficient that defines the number of repeated $\mathcal{H}_{0}$ decisions that will activate the power reduction mechanism of the estimate.

\section{Simulation results}

The evaluation of the sensing algorithms is realized with the use of the receiver operating characteristic (ROC) curves. These figures depict the tradeoff between the $P_{\mathrm{FA}}$ and the $P_{\mathrm{D}}$, as well as the relationship between the $P_{\mathrm{D}}$ or the $P_{\mathrm{FA}}$ vs. the SNR. The specifications of the system that was used for simulation are presented in Table 1.

In order to have a reference point during the evaluation, an equivalent - commonly used - detector was implemented: the energy detector for a single primary channel. This energy detector does not use the filter bank at all. It covers a specific primary channel (that is spanned by $L=4$ or 8 filter paths) and accumulates the incoming

Table 1 Simulation parameters

\begin{tabular}{|c|c|}
\hline & Values/description \\
\hline $\begin{array}{l}\text { Number of subchannels of the filter } \\
\text { bank over the entire bandwidth }\end{array}$ & 32 \\
\hline $\begin{array}{l}\text { Number of primary channels over } \\
\text { the entire bandwidth }\end{array}$ & 8 or 4 \\
\hline $\begin{array}{l}\text { Number of subchannels per } \\
\text { primary channel }\end{array}$ & 4 or 8 \\
\hline Coefficients for the prototype filter & 101 \\
\hline Roll-off for the prototype filter & 0.8 \\
\hline Noise & AWGN \\
\hline Primary transmission & $\begin{array}{l}\text { White Gaussian random } \\
\text { variable; single carrier } \\
\text { transmission }\end{array}$ \\
\hline Filter type for the Uniform DFT & Root Nyquist \\
\hline $\begin{array}{l}\text { Design algorithm for the DFT filter } \\
\text { bank }\end{array}$ & {$[40]$} \\
\hline $\begin{array}{l}\text { Filter type in the OFDM-OQAM } \\
\text { receiver }\end{array}$ & MDFT with PR \\
\hline $\begin{array}{l}\text { Design algorithm for the MDFT filter } \\
\text { bank }\end{array}$ & {$[41]$} \\
\hline Radio channels & $\begin{array}{l}\text { Rayleigh paths with } \\
\text { exponential PDP }\end{array}$ \\
\hline
\end{tabular}

signal (or noise) energy. In order to fairly compare the filter bank-based sensor and the energy detector, the following matching is made. Since the filter bank collects $N$ samples from each of the $L$ subchannels that span the primary channel, the equivalent energy detector will collect $L N$ samples directly from the PS channel. Calculation of the thresholds and the theoretical estimation of the $P_{\mathrm{FA}}$ and $P_{\mathrm{D}}$ is made through the well-known energy detector distributions [24].

At first, the ROC curve $\left(P_{\mathrm{D}}\right.$ vs. $\left.P_{\mathrm{FA}}\right)$ for a system with $M=32, L=4$ and $N=8$ is presented in Figure 7 . As expected, the optimum detectors provide the best $P_{\mathrm{D}}$ vs. $P_{\mathrm{FA}}$ tradeoff. Moreover, since both optimum detectors (for uniform DFT and MDFT filter banks) have identical distributions, their performance is very similar. A very detailed observation of the curve will show that the MDFT filter bank performs slightly better. This is due to the fact that the matrix inversions and multiplications for the uniform DFT metric may result in small precision error.

On the other hand, the weighted energy detector practically fills the gap between the optimal detectors and the equivalent PS channel energy detector. It is noted that the weighted energy detector performs satisfactorily and presents stability.

Another conclusion that can be derived is that the theoretical calculations fit the simulation results exceptionally. It must be noted that the used radio channel responses were produced using a model with exponential PDP $(\lambda=$ $0.5,24$ coefficients). Hence, although the channel impulse responses were not ideally constructed to fit the approximately flat radio channel per subchannel approximation, the detector performed as predicted with high accuracy. On the other hand, the radio channel frequency selectivity acted destructively for the energy detector that monitors the entire wideband primary channel and degraded its performance.

In Figure 9, the respective figure for $P_{\mathrm{D}}$ vs. SNR for the same scenario is also presented. Figure 9 supports the aforementioned concluding remarks and confirms the superiority of the optimal detectors over the SNR values.

In order to better understand and evaluate the performance of the detectors over frequency selective channels, the following test was performed. The configuration of Table 1 was assumed with four PS channels, eight filters per subchannel and $N=4$. Channels with exponential PDP were tested, and the parameter $\lambda$ was used as a measure of frequency selectivity, since small $\lambda$ value means large delay spread and consequently high frequency selectivity. In case the approximately flat radio channel per subchannel assumption is true, the estimated $P_{\mathrm{D}}$ value from the simulation tests is expected to coincide with the theoretically calculated value. Otherwise, deviation from the theoretical value will occur. During the simulation campaign, $\lambda$ varied from 0.2 to $3\left(C_{\max }=48\right.$ 
ROC curves ( $P_{D}$ vs SNR) from Simulation Results and Theoretic Analysis for the Filterbank Detectors for Observation Vector length 8 $\left(P_{F A}=0.05\right.$, filters/primary channel $=4$, detector lag per filter $=8$ samples

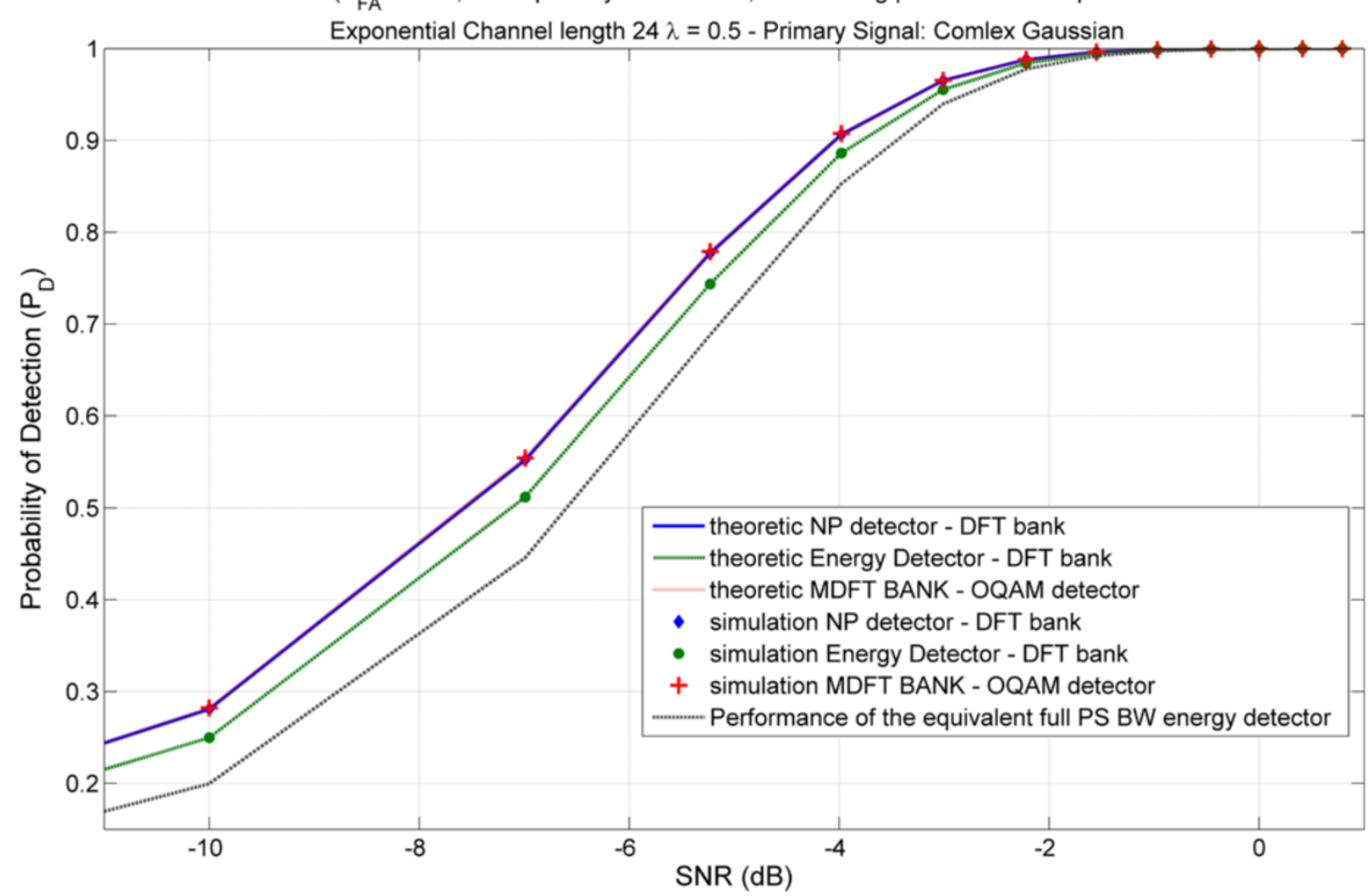

Figure 9 ROC curve $P_{D}$ vs. SNR for detection under AWGN.

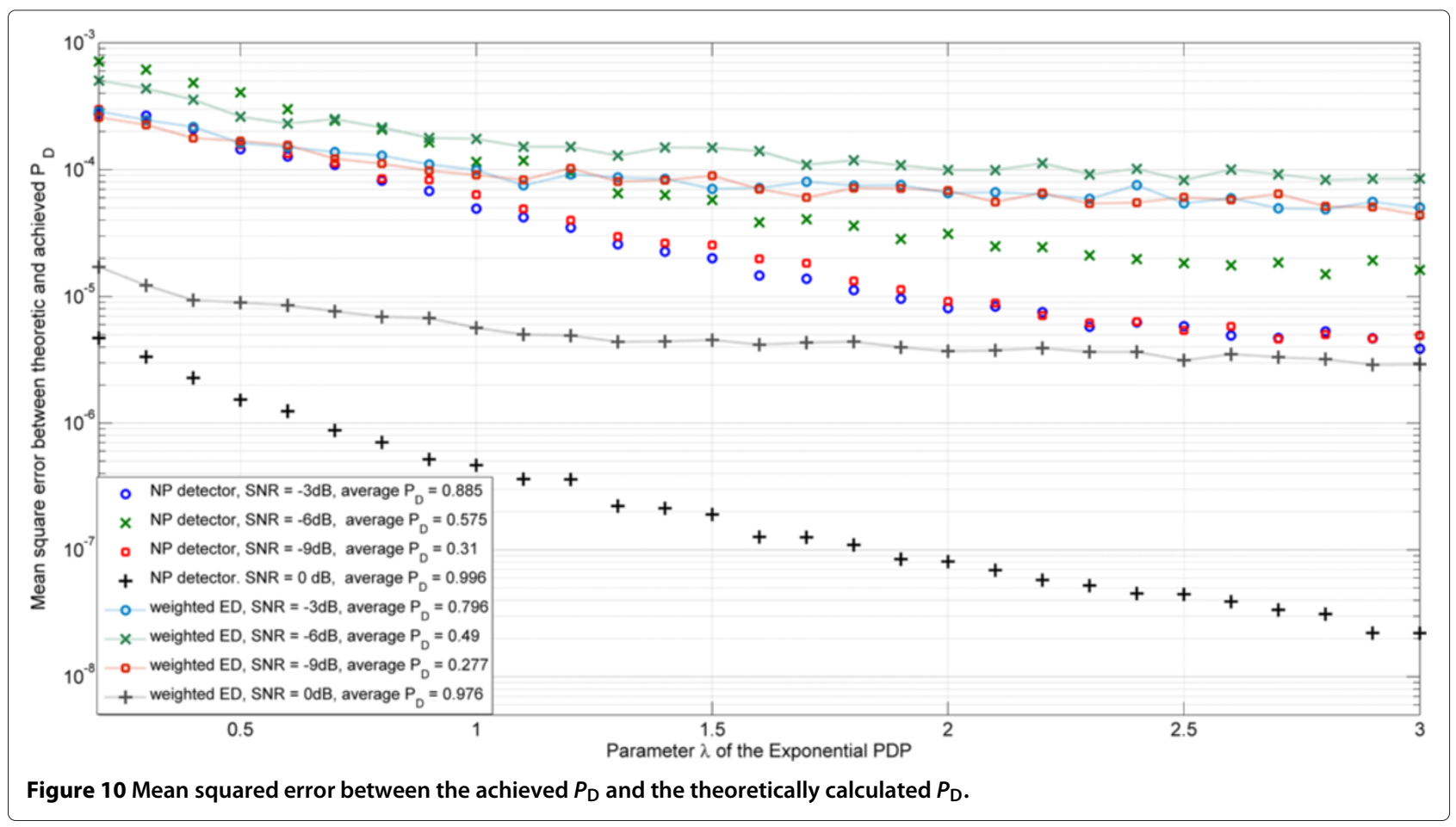


paths for all cases). For each $\lambda$ and for $\mathrm{SNR}=0.125,0.25$, 0.5 and 1 , a set of 4,000 channels was produced and the mean squared error between the theoretically calculated and the estimated via simulation $P_{\mathrm{D}}$ was calculated. The target $P_{\mathrm{FA}}$ was set to 0.1 . The results are presented in Figure 10. It is noted that for presentation reasons, since the results for the optimum detectors are very similar, only the mean square $P_{\mathrm{D}}$ error for the OFDM-OQAM detector is provided.

The following conclusions can be derived. The error increases with frequency selectivity (as $\lambda$ decreases); however, in every case, even when the frequency selectivity is significantly high (e.g. $\lambda=0.2$ ), the mean square error remains lower than 0.001 . Therefore, it is concluded that even in cases where the 'approximately flat' assumption is not valid, the segmentation of the signal in several subchannels $(L=8)$ using a filter bank acts beneficially for the spectrum sensing algorithm. Moreover, the error depends on the SNR and more particularly on the achieved $P_{\mathrm{D}}$ value for the specific SNR. It is observed that as the $P_{\mathrm{D}}$ value approaches 0 or 1 , the mean square error decreases significantly (e.g. $\mathrm{SNR}=0 \mathrm{~dB}$ ). Error maximization is achieved for the SNR values that correspond to a probability of detection near $P_{\mathrm{D}}=0.5$ $(\mathrm{SNR}=0.25)$. SNRs that provide intermediate $P_{\mathrm{D}}$ values may have similar error values $(\mathrm{SNR}=0.125$ and 0.5$)$. A significant observation of the simulation tests is that the $P_{\mathrm{D}}$ error is biased. This means that the achieved $P_{\mathrm{D}}$ will be lower than the theoretically calculated, as expected, since the proposed detectors are optimum. Therefore, the mean square error of the $P_{\mathrm{D}}$ can be used as a measure of performance degradation of the detector due to the deviation of the radio channel from the approximately flat assumption.

The weighted energy detector presents similar behaviour. However, it can be seen that the error floor, as $\lambda$ increases, is higher than the one achieved by the NP detectors. Moreover, the error reduction slope is significantly smaller, which means that despite the fact that error

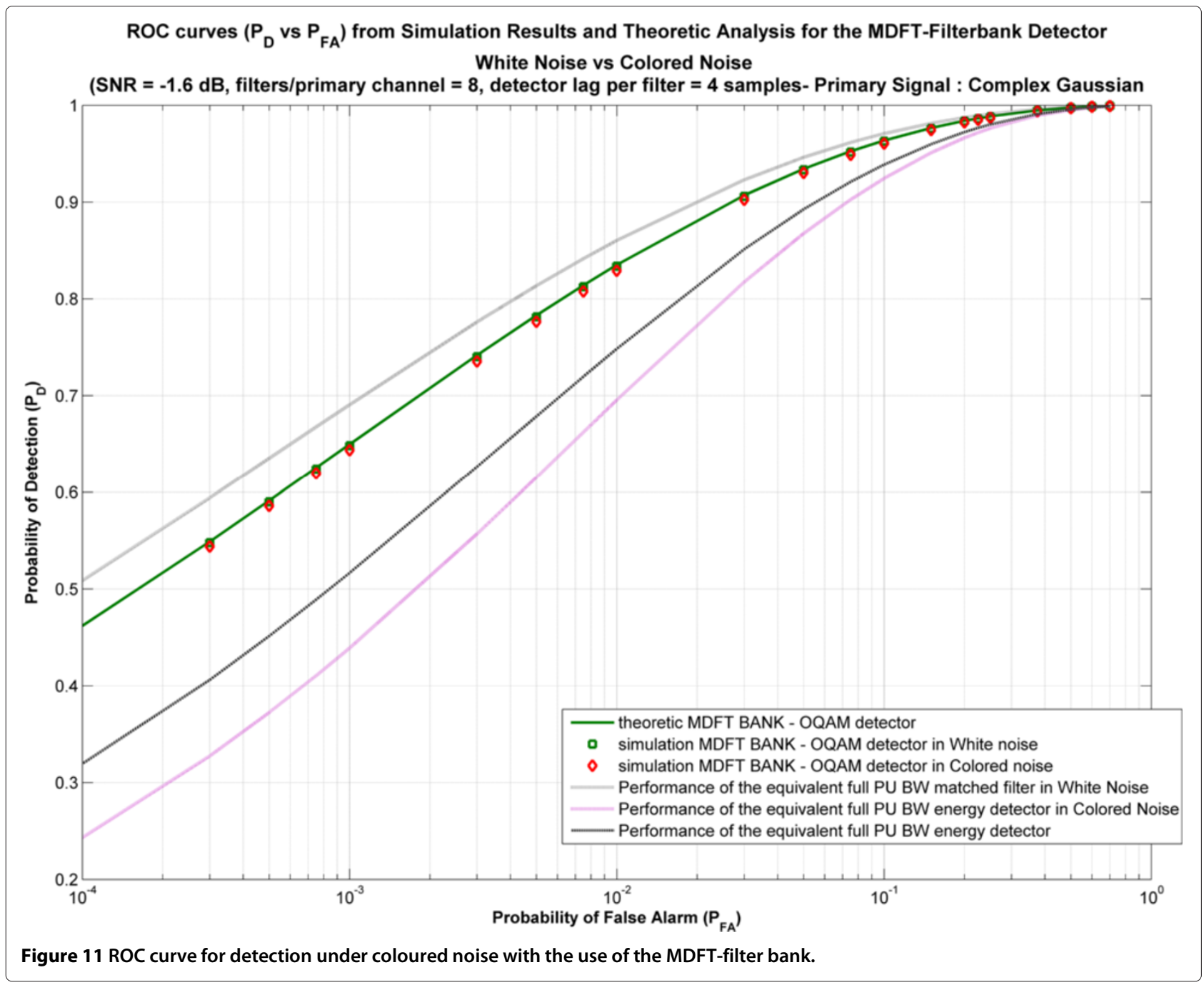


values are generally higher, the weighted energy detector is not significantly affected by frequency selectivity. In addition, for low $\lambda$ values (high frequency selectivity) and with the exception of $\mathrm{SNR}=0 \mathrm{~dB}$, the mean square error for the weighted energy detector becomes less or equal to the error values of the NP detectors. The exception for $\mathrm{SNR}=0 \mathrm{~dB}$ can be justified by the fact that the average $P_{\mathrm{D}}$ value is not that close to unity as in the case of the NP detector (0.996), and therefore the error levels are higher for the whole range of $\lambda$ values. It can be concluded that the weighted energy detector has a higher (biased) error floor; however, it is more resilient in frequency selective environments, as far as the $P_{\mathrm{D}}$ error is concerned. It is also noted that for all the studied cases, since AWGN was assumed, the target $P_{\mathrm{FA}}=0.1$ that depends only on the noise distribution was achieved with great accuracy.

The operation of the optimum detector under coloured noise is evaluated in Figures 11 and 12, where the number of samples per subchannel was set to 4 and $\mathrm{SNR}=-1.6 \mathrm{~dB}$. In Figure 12, the targeted $P_{\mathrm{FA}}$ was set to 0.05 .

Noise becomes frequency selective with the same procedure that models the Gaussian incoming PS signal. Hence, before entering the filter bank, the noise samples are filtered with a predefined impulse response. In the results presented in Figures 11 and 12, Rayleigh channels with exponential PDP $\left(\lambda_{\text {noise }}=1.6, \lambda_{\text {signal }}=1\right)$ were used for the generation of the noise and signal vector respectively. This scenario practically simulates a remote interferer or a different $C R$ transmission.

In Figures 11 and 12, the MDFT Neyman-Pearson detector performance is depicted, since the optimal uniform DFT detector performs similarly. Moreover, the performance of the detector under white noise is also presented. It is noticed that the filter bank detector in the existence of coloured noise follows quite accurately the performance of the white noise detector due to the fact that coloured noise support can be seamlessly integrated in the optimum detector. The small degradation is caused from the increase of the overall ambiguity due to the frequency selectivity of the noise.

On the other hand, the degradation of the equivalent PS channel energy detector is quite noticeable. The lack of robustness under coloured noise is typical in many common and popular detectors. At the same time, the detector fails to achieve the desired $P_{\mathrm{FA}}$ levels. Since the noise is correlated, the distribution used for threshold calculation by the energy detector is wrong, causing degradation in the $P_{\mathrm{FA}}$ performance. A similar phenomenon does not appear in the filter bank sensor. In Figures 11 and 12, the performance of the matched filter detector for the PS channel is also depicted as an upper limit for the performance of the detection schemes.

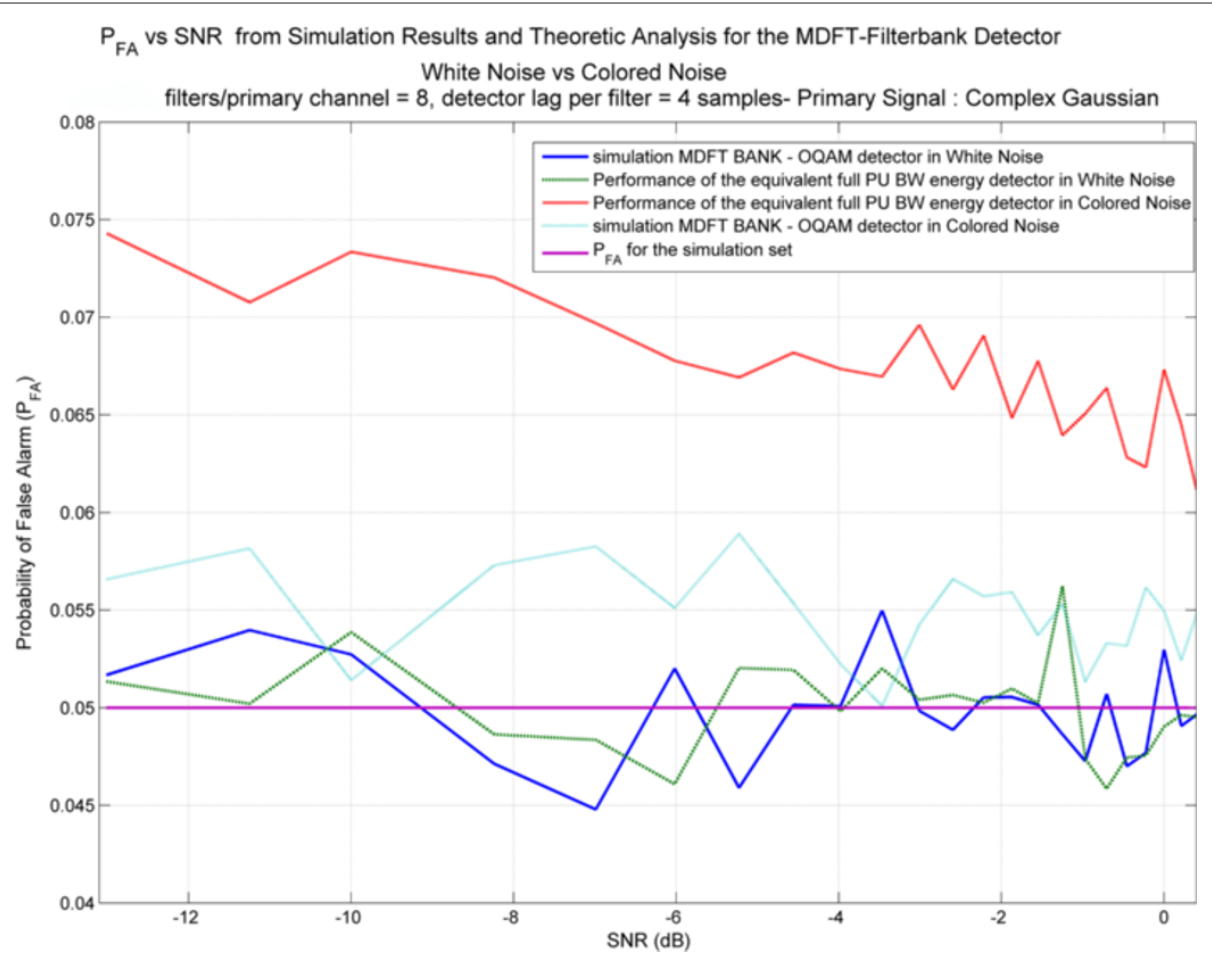

Figure $12 P_{\mathrm{FA}}$ vs. SNR with the use of four samples per observation vector per subchannel. 


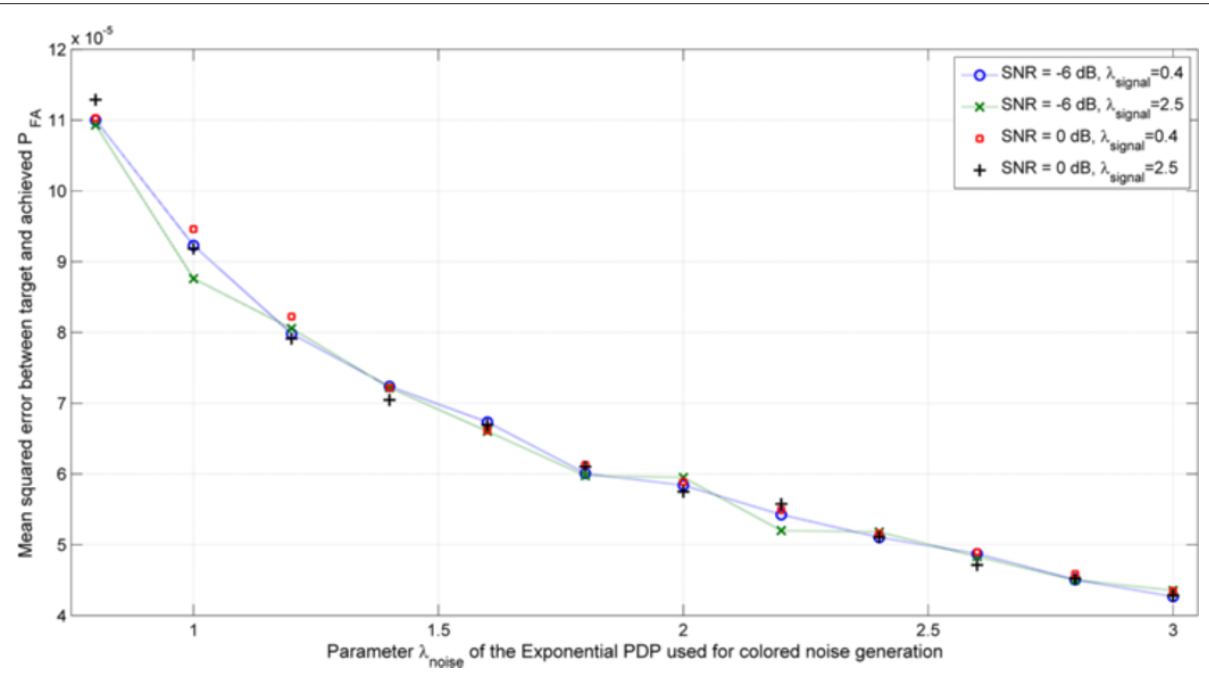

Figure 13 Mean squared error between achieved $P_{\mathrm{FA}}$ and target $P_{\mathrm{FA}}$ for coloured noise.

In order to quantify the effect of frequency selectivity on the NP detector with coloured noise and compare it with the AWGN detector, extended simulation tests were performed. A significant difference in the performance of the detector over coloured noise compared with the equivalent AWGN detector is the fact that there is an error in the achieved $P_{\mathrm{FA}} . P_{\mathrm{FA}}$ error was not present in the AWGN detector, since the distribution of the metric under $\mathcal{H}_{0}$ was accurate and it was not affected by the frequency selectivity of the signal. However in this case, the approximately flat per subchannel assumption is also used for the statistical description of the coloured noise vector, and it is expected to affect the $P_{\text {FA }}$ performance of the detector. During the simulation campaign, $\lambda_{\text {signal }}$ (the exponential factor of the PDP for the signal) varied from 0.2 to 3 , while $\lambda_{\text {noise }}$ (the exponential factor of the PDP for the noise) varied from 0.8 to 3 . For each pair of parameters, a set of 2,000 channels was produced and the estimated via simulation $P_{\mathrm{FA}}$ was calculated and compared with the target $P_{\mathrm{FA}}=0.1$. The results are presented in Figure 13 .

In Figure 13, it becomes clear that since the metric under $\mathcal{H}_{0}$ is not affected by the validity of the approximately flat assumption of the PS signal, the $P_{\mathrm{FA}}$ error does not depend on $\lambda_{\text {signal. }}$ Simulation results also indicate that the $P_{\mathrm{FA}}$ error does not depend on the SNR since lower SNR values may indicate more powerful noise; however, they do not affect the adequacy of the approximately flat assumption for the noise vector. On the other hand,

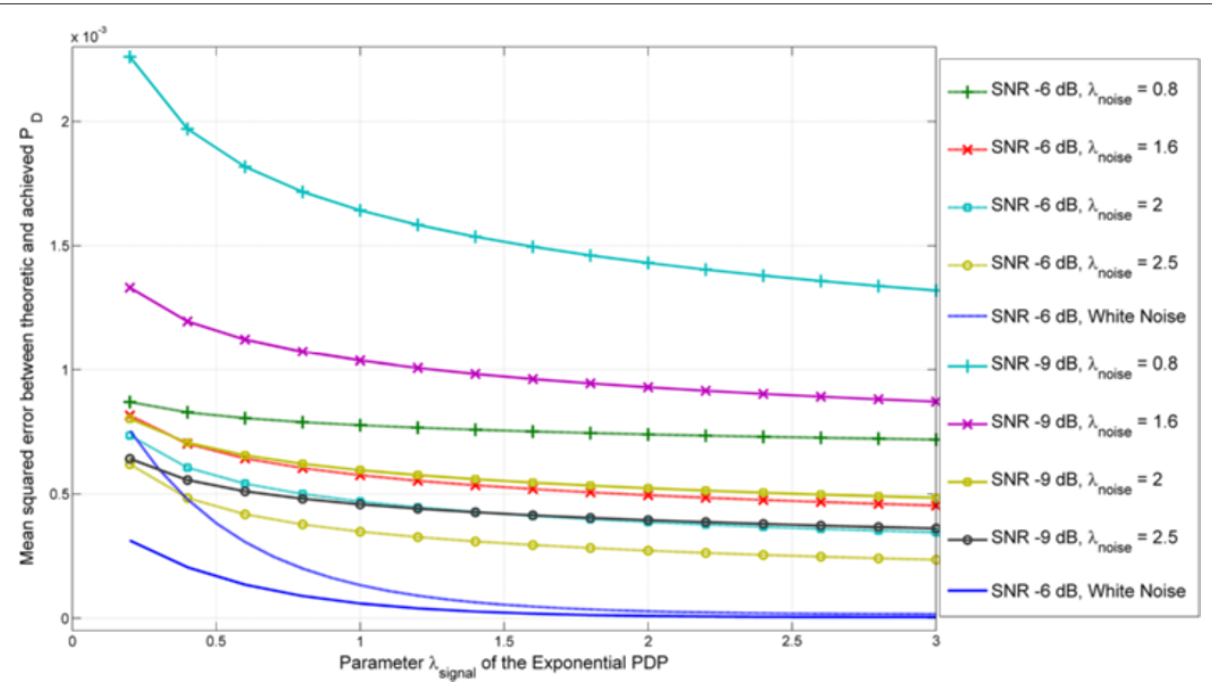

Figure 14 Mean squared error between the achieved $P_{D}$ and the theoretically calculated $P_{D}$ for coloured noise. 

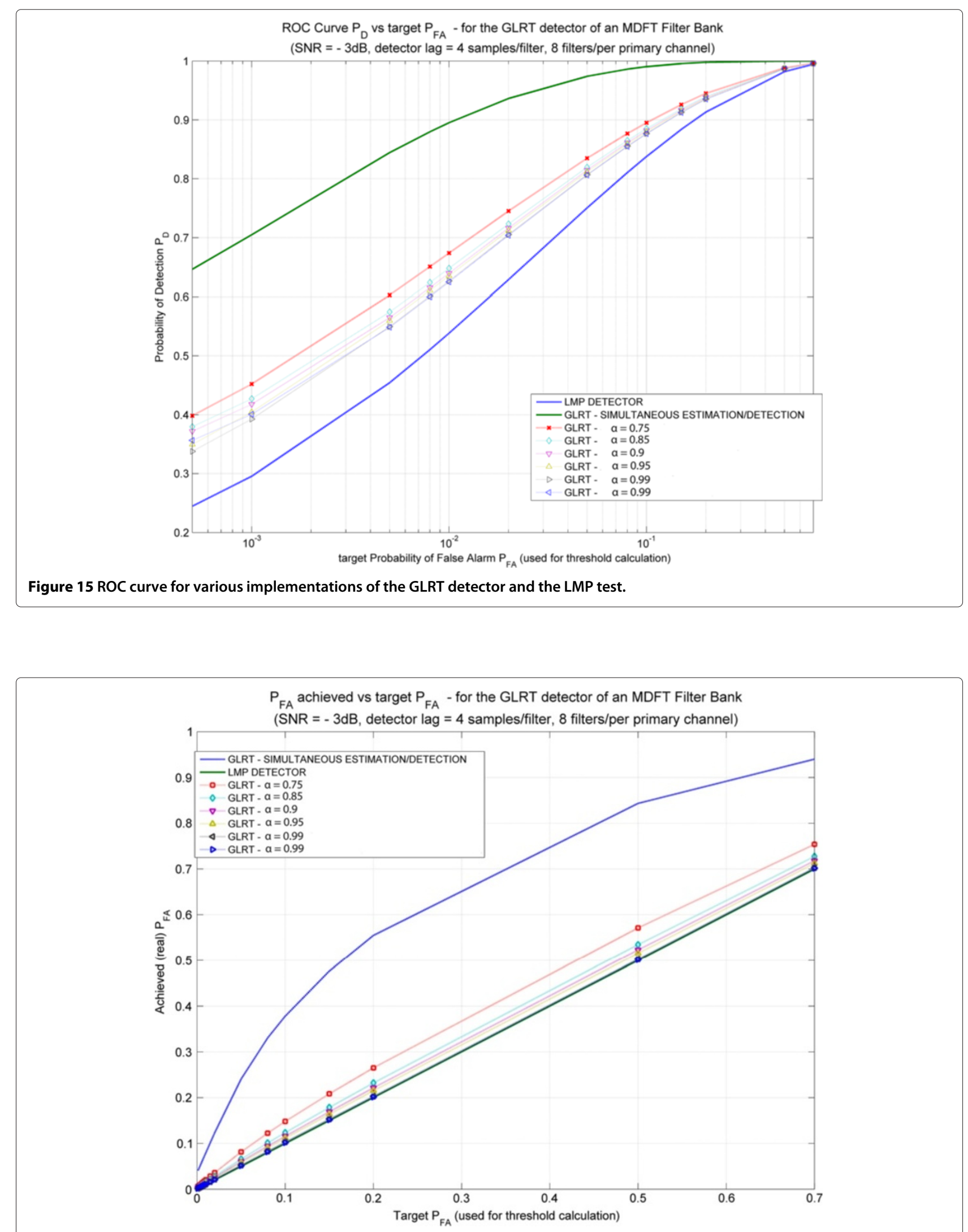

Figure 16 Simulated $P_{\mathrm{FA}}$ vs. targeted $P_{\mathrm{FA}}$ for various implementations of GLRT and LMP detectors. 
$P_{\mathrm{FA}}$ error depends on the parameter $\lambda_{\text {noise }}$ that is used to colour the noise. Lower $\lambda_{\text {noise }}$ values indicate strongly selective noise and thus the $P_{\mathrm{FA}}$ error increases due to deviation from the approximately flat per subchannel assumption. It is seen that e.g. for $\lambda_{\text {noise }}=0.8$, the mean square error is larger than 0.0001 which means that the deviation from the target $P_{\mathrm{FA}}$ may be higher than $1 \%$. This deviation may significantly affect the $P_{\mathrm{D}}$ performance of the detector as presented in Figure 14.

In Figure 14, it can be seen that the mean square error between the achieved $P_{\mathrm{D}}$ and the theoretically calculated $P_{\mathrm{D}}$ in most cases is significantly increased compared with the AWGN scenario. Moreover, the $P_{\mathrm{D}}$ error now increases as the SNR is reduced in contrast with the AWGN case where the error depended on the $P_{\mathrm{D}}$ value for a given SNR. This happens due to the fact that as the SNR decreases, the overlap of the distributions under $\mathcal{H}_{0}$ and $\mathcal{H}_{1}$ grows. The $P_{\mathrm{FA}}$ error practically moves the decision threshold. When the distribution overlap is large, the produced $P_{\mathrm{D}}$ error due to the threshold offset will also be larger. It is also noticed that when noise frequency selectivity increases, $P_{\mathrm{D}}$ error also increases due to the fact that under $\mathcal{H}_{1}$, the metric distribution is affected by the validity of the approximately flat assumption for both PS signal and noise. An exception in the comparison of $P_{\mathrm{D}}$ error over coloured and white noise is observed for $\mathrm{SNR}=-6 \mathrm{~dB}$ when $\lambda_{\text {signal }}$ is lower than 0.4 and $\lambda_{\text {noise }}=2.5$. Figure 10 shows that error was maximized for $\mathrm{SNR}=-6 \mathrm{~dB}$. During the simulation tests for coloured noise, it was shown that the $P_{\mathrm{FA}}$ error for high $\lambda_{\text {noise }}$ values is biased and the achieved $P_{\mathrm{FA}}$ will be actually smaller than the targeted. Smaller $P_{\mathrm{FA}}$ means increase of the $P_{\mathrm{D}}$ value. This increase of the $P_{\mathrm{D}}$ value counteracts with the biased $P_{\mathrm{D}}$ error due to PS signal frequency selectivity. Therefore, in the specific case, the $P_{\mathrm{D}}$ error of the coloured noise detector is smaller than the AWGN case.

Finally, in Figures 15, 16, 17, the ROC curves that evaluate the performance of the GLRT detector are presented. Various versions of the GLRT are implemented. In the first version, estimation and detection are performed over the same observation vector. In the rest of the cases, the detection is performed using previous estimation results with a varying forgetting factor (from 0.75 to 0.99 ). The

ROC Curve $P_{F A}$ vs SNR - for the GLRT detector of an MDFT Filter Bank (SNR = - 3dB, detector lag = 4 samples/filter, 8 filters/per primary channel)

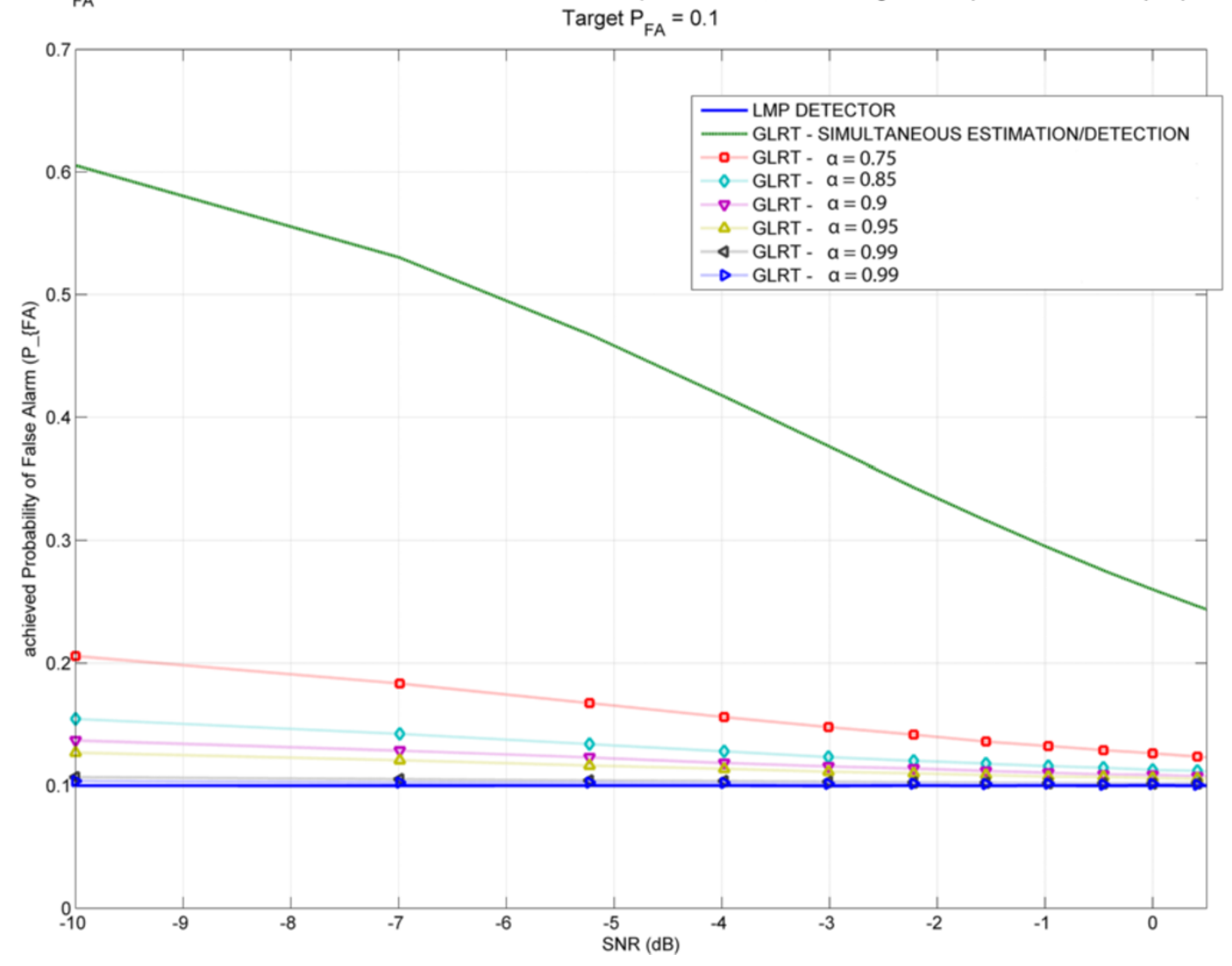

Figure 17 Simulated $P_{\mathrm{FA}}$ vs. the SNR value for targeted $P_{\mathrm{FA}}=0.1$ for various implementations of GLRT and LMP detectors. 
GLRT performance is compared with the LMP test. All detectors are applied to the system of Table 1 using four samples per subchannel as the observation vector and eight subchannels per PS signal.

From Figure 15, it is noticed that the memoryless GLRT achieves greater $P_{\mathrm{D}}$. However, this result is misleading. In Figures 16 and 17, it can be seen that not only the memoryless GLRT, but also GLRT implementations with short memory, fail to achieve the target $P_{\mathrm{FA}}$ that is defined by the Neyman-Pearson optimum detector. Therefore, it is concluded that the GLRT is generally biased towards the positive outcome $\mathcal{H}_{1}$. These figures also indicate that the main disadvantage of the GLRT detector is its weakness to follow the targeted $P_{\mathrm{FA}}$.

It is clear that the $P_{\mathrm{FA}}$ value achieved by the memoryless GLRT is heavily diverged by the targeted $P_{\mathrm{FA}}$. As the SNR increases, the actual $P_{\mathrm{FA}}$ seems to asymptotically approach the targeted value. The LMP, by definition, complies accurately with the required targeted SNR, but on the other hand it is lacking in $P_{\mathrm{D}}$ performance. The GLRT implementations with memory converge relatively fast to a value that it is close to the desired $P_{\mathrm{FA}}$, while they maintain higher $P_{\mathrm{D}}$ values compared to the LMP. Increase of the forgetting factor over 0.95 does not provide significant improvement.

\section{Conclusions}

This paper provided the theoretical framework for the analysis and design of filter bank-based detectors for spectrum sensing applications in cognitive radios. The optimal detectors for uniform DFT and modified DFT filter banks were developed and evaluated. Moreover, the simple but non-optimal weighted energy detector for uniform DFT filter banks was analysed. The filter-bankbased sensing techniques were proved (through simulations) robust under coloured noise. In addition, they exploit the radio channel frequency selectivity to improve their performance. Finally, implementable sensors using the Locally Most Powerful and the Generalized Likelihood Radio tests were proposed and evaluated through simulation.

\section{Competing interests}

The authors declare that they have no competing interests.

\section{Acknowledgements \\ The research is co-financed by the European Union (European Social Fund-ESF) and Greek national funds through the Operational Program 'Education and Lifelong Learning' of the National Strategic Reference Framework (NSRF)-Research funding Program: THALES Investing in knowledge society through the European Social Fund, MIS379489.}

Received: 12 December 2013 Accepted: 1 May 2014

Published: 3 June 2014

\section{References}

1. S Haykin, Cognitive radio: brain-empowered wireless communications. IEEE J. Selected Areas Commun. 23(2), 201-220 (2005)
2. TYucek, H Arslan, A survey of spectrum sensing algorithms for cognitive radio applications. IEEE Commun. Surv. Tutorials. 11(1), 116-130 (2009). doi:10.1109/SURV.2009.090109

3. A Huseyin, Cognitive Radio, Software Defined Radio, and Adaptive Wireless Systems. (Springer, New York, 2007)

4. B Farhang-Boroujeny, Filter bank spectrum sensing for cognitive radios. IEEE Trans. Signal Process. 56(5), 1801-1811 (2008)

5. B Farhang-Boroujeny, R Kempter, Multicarrier communication techniques for spectrum sensing and communication in cognitive radios. IEEE Comm. Mag. 46(4), 80-85 (2008)

6. D Jinfeng, S Signell, Classic OFDM systems and pulse shaping OFDM/OQAM systems. Technical Report 07:01, KTH, Electronic, Computer and Software Systems, ECS (2007)

7. S Kai, Z Weiwei, W Guangyu, Design and implementation of MDFT filter bank based multicarrier modulation systems, in 2013 IEEE International Conference on Signal Processing, Communication and Computing (ICSPCC), (2013), pp. 1-6. doi:10.1109/ICSPCC.2013.6663968

8. K-H Chen, T-D Chiueh, A cognitive radio system using discrete wavelet multitone modulation. IEEE Trans. Circ. Syst. Regular Pap. 55(10), 3246-3258 (2008)

9. G Cherubini, E Eleftheriou, S Olcer, Filtered multitone modulation for very high-speed digital subscriber lines. IEEE J. Sel. Area Comm. 20(5), 1016-1028 (2002)

10. M Bellanger, $T$ Ihalainen, M Renfors, Filter bank based cognitive radio physical layer, in ICT-MobileSummit 2009 Conference Proceedings (Santander, 10-12 June 2009)

11. DJ Thomson, Spectrum estimation and harmonic analysis. Proc. IEEE 70(9), 1055-1096 (1982)

12. M Kim, J-i Takada, Efficient multi-channel wideband spectrum sensing technique using filter bank, in 2009 IEEE 20th International Symposium on Personal, Indoor and Mobile Radio Communications, (2009), pp. 1014-1018. doi:10.1109/PIMRC.2009.5450175

13. MA Lagunas, Al Perez-Neira, MA Rojas, Spectrum sensing for cognitive radio in filter-bank multi-carrier communications systems: the candidate estimate, in Proceedings of the International Conference on Circuits, Systems, Signals (CSS) (Malta, 15-17 September 2010)

14. H Cao, W Jiang, T Kaiser, Multi-channel robust spectrum sensing with low-complexity filter bank realization, in 2013 IEEE 24th International Symposium on Personal Indoor and Mobile Radio Communications (PIMRC) (London, 8-11 September 2013), pp. 861-865

15. R Mahesh, AP Vinod, C Moy, J Palicot, A low complexity reconfigurable filter bank architecture for spectrum sensing in cognitive radios, in $3 r d$ International Conference on Cognitive Radio Oriented Wireless Networks and Communications, CrownCom, Singapore, 15-17 May 2008, pp. 1-6

16. M Lin, AP Vinod, CMS See, Very low complexity variable resolution filter banks for spectrum sensing in cognitive radios using multi-stage coefficient decimation, in 5th International Conference on Wireless Communications, Networking and Mobile Computing, WiCom '09 (Beijing, 24-26 September 2009), pp. 1-4

17. SJ Darak, H Zhang, J Palicot, AP Vinod, Efficient spectrum sensing for green cognitive radio using low complexity reconfigurable fast filter bank, in 2013 International Conference on Advanced Technologies for Communications (ATC) (Pyeong-chang, 27-30 January 2013), pp. 318-322

18. M Narendar, AP Vinod, AS Madhukumar, AK Krishna, A tree-structured $\{D F T\}$ filter bank based spectrum detector for estimation of radio channel edge frequencies in cognitive radios. Phys. Commun. 9(0), 45-60 (2013). doi:10.1016/j.phycom.2013.06.001

19. M Lin, AP Vinod, C See, A new flexible filter bank for low complexity spectrum sensing in cognitive radios. J. Signal Process. Syst. 62(2), 205-215 (2011)

20. T Sato, M Umehira, A new spectrum sensing scheme using overlap FFT filter-bank for dynamic spectrum access, in 2011 Sixth International ICST Conference on Cognitive Radio Oriented Wireless Networks and Communications (CROWNCOM), Osaka, 1-3 June 2011, pp. 6-10

21. T Hunziker, UU Rehman, D Dahlhaus, Spectrum sensing in cognitive radios: design of DFT filter banks achieving maximal time-frequency resolution, in 2011 8th International Conference on Information, Communications and Signal Processing (ICICS), (2011), pp. 1-5. doi:10.1109/ICICS.2011.6173530

22. S Fahmy, L Doyle, ed. by P Sirisuk, F Morgan, T El-Ghazawi, and Amano H, Reconfigurable polyphase filter bank architecture for spectrum sensing, 
in Reconfigurable Computing: Architectures, Tools and Applications. Lecture Notes in Computer Science, vol. 5992 (Springer, Berlin, 2010), pp. 343-350

23. KG Smitha, AP Vinod, A multi-resolution fast filter bank for spectrum sensing in military radio receivers. IEEE Trans. Very Large Scale Integration (VLSI) Syst. 20(7), 1323-1327 (2012). doi:10.1109/TVLSI.2011.2151214

24. SM Kay, Fundamentals of Statistical Signal Processing, Detection Theory. (Prentice Hall, New Jersey, 1998)

25. H Al-Hmood, RS Abbas, A Masrub, HS Al-Raweshidy, An estimation of primary user's SNR for spectrum sensing in cognitive radios, in 2013 Third International Conference on Innovative Computing Technology (INTECH) (London, 29-31 August 2013), pp. 479-484

26. S Dikmese, M Renfors, Optimized FFT and filter bank based spectrum sensing for Bluetooth signal, in 2012 IEEE Wireless Communications and Networking Conference (WCNC) (Paris, 1-4 April 2012), pp. 792-797

27. F Harris, Multirate Signal Processing for Communication Systems. (Prentice Hall, New Jersey, 2004)

28. PP Vaidyanathan, Multirate Systems and Filter Banks. (Prentice Hall, New Jersey, 1993)

29. T Karp, NJ Fliege, Modified DFT filter banks with perfect reconstruction. IEEE Trans. Circuits Syst. II: Analog Digit. Signal Process. 46(11), 1404-1414 (1999)

30. J Proakis, M Dimitris, Digital Signal Processing - Principles, Algorithms and Applications, 4th edn. (Prentice Hall, New Jersey, 2007)

31. IS Bendat, AG Piersol, Random Data, Analysis and Measurement Procedures. (Wiley Interscience, New York, 1985)

32. GAF Seber, A Matrix Handbook for Statisticians. (Wiley, New York, 2007)

33. JR Silvester, Determinants of block matrices. Math. Gaz. 501, 460-467 (2000)

34. A Papoulis, SU Pillai, Random Variables and Stochastic Processes. (McGraw-Hill Education, New York, 2001)

35. EW Weisstein, CRC Concise Encyclopedia of Mathematics, 2nd edn. (Chapman and Hall - CRC, Florida, 2000), pp. 410-411

36. M-S Alouini, A Abdi, M Kaveh, Sum of gamma variates and performance of wireless communication systems over Nakagami-fading channels. IEEE Trans. Veh Tech. 50(6), 1471-1480 (2001)

37. N Balakrishnan, Continuous Multivariate Distributions. Encyclopedia of Statistical Sciences. (Wiley, Hoboken, 2001)

38. GH Golub, V Loan, Matrix Computations, 2nd edn. (Johns Hopkins University, Baltimore, 1989)

39. V Poor, An Introduction to Signal Detection and Estimation. (Springer, New York, 1994)

40. T Parks, J McClellan, A program for the design of linear phase finite impulse response digital filters. IEEE Trans. Audio Electroacoust. 20(3), 195-199 (1972)

41. HXu, W-S Lu, A Antoniou, Efficient iterative design method for cosine-modulated QMF banks. IEEE Trans. Signal Process. 44(7), 1657-1668 (1996)

doi:10.1186/1687-6180-2014-82

Cite this article as: Maliatsos et al.: Elaborate analysis and design of filter-bank-based sensing for wideband cognitive radios. EURASIP Journal on Advances in Signal Processing 2014 2014:82.

\section{Submit your manuscript to a SpringerOpen ${ }^{\odot}$ journal and benefit from:}

- Convenient online submission

- Rigorous peer review

- Immediate publication on acceptance

- Open access: articles freely available online

- High visibility within the field

- Retaining the copyright to your article

Submit your next manuscript at $\boldsymbol{\wedge}$ springeropen.com 